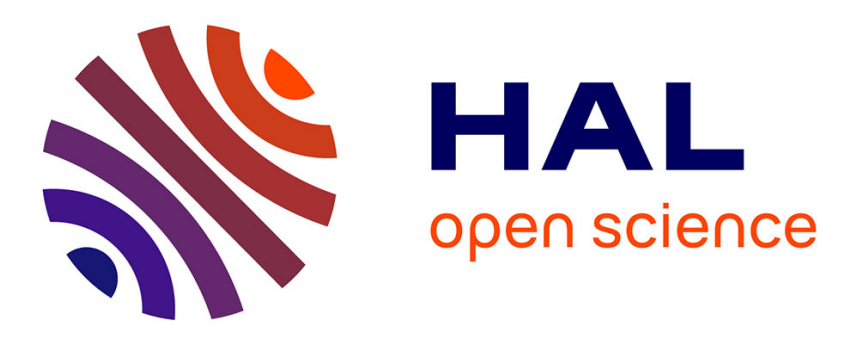

\title{
Kinetics and mechanisms of stress relaxation in sputtered silver thin films
}

\author{
Quentin Hérault, Iryna Gozhyk, Matteo Balestrieri, Hervé Montigaud, Sergey \\ Grachev, Remi Lazzari
}

\section{- To cite this version:}

Quentin Hérault, Iryna Gozhyk, Matteo Balestrieri, Hervé Montigaud, Sergey Grachev, et al.. Kinetics and mechanisms of stress relaxation in sputtered silver thin films. Acta Materialia, 2021, pp.117385. 10.1016/j.actamat.2021.117385 . hal-03381625

\section{HAL Id: hal-03381625 https://hal.science/hal-03381625}

Submitted on 17 Oct 2021

HAL is a multi-disciplinary open access archive for the deposit and dissemination of scientific research documents, whether they are published or not. The documents may come from teaching and research institutions in France or abroad, or from public or private research centers.
L'archive ouverte pluridisciplinaire HAL, est destinée au dépôt et à la diffusion de documents scientifiques de niveau recherche, publiés ou non, émanant des établissements d'enseignement et de recherche français ou étrangers, des laboratoires publics ou privés. 


\section{Contents}

1 Introduction $\quad 3$

2 Experimental $\quad 4$

3 Mechanisms of stress relaxation after continuous deposition $\quad 6$

3.1 The three exponential components of stress relaxation . . . . . . 7

3.2 The thermal stress component and the heat exchange model . . . 11

3.3 Temperature evolution during sputtering deposition and relaxation 11

3.4 Identifying the thermal stress component . . . . . . . . . . . . . 14

3.5 Assignment of the other relaxation mechanisms . . . . . . . . . 17

3.6 Coupling between atom diffusion at GBs and GB grooving in stress relaxation ................... 20

3.7 Stress gradient along GBs and GB grooving dynamics . . . . . . 22

4 Stress relaxation after interrupted deposition $\quad 25$

5 Stress relaxation in non-percolated films $\quad 27$

6 Conclusion 28

7 Acknowledgements $\quad 29$

Graphical Abstract

Kinetics and mechanisms of stress relaxation in sputtered silver thin films

Quentin Hérault, Iryna Gozhyk, Matteo Balestrieri, Hervé Montigaud, Sergey Grachev, Rémi Lazzari

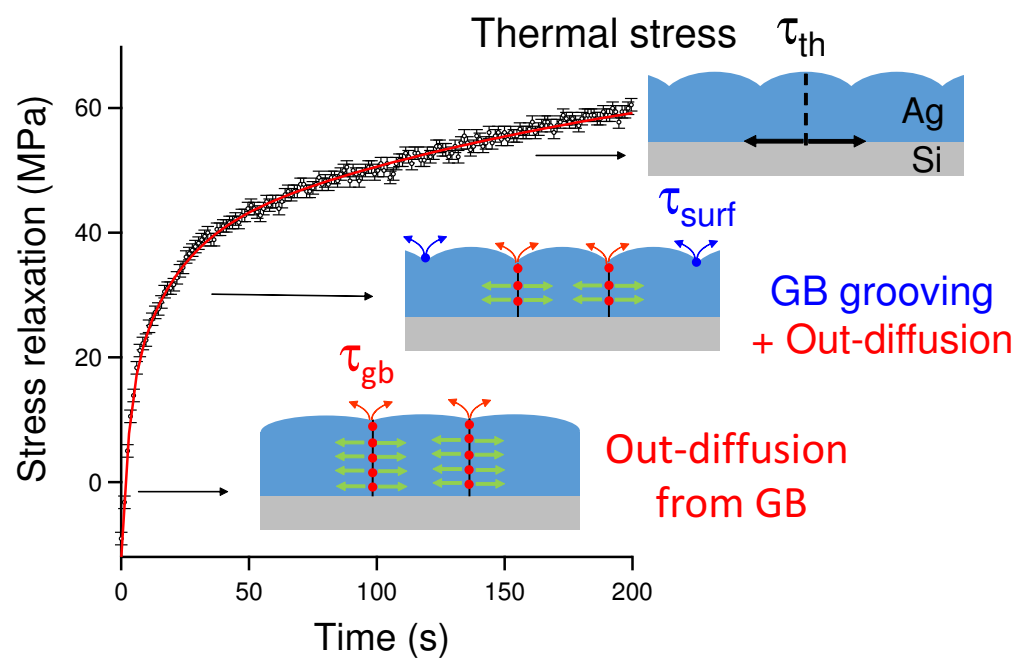




\title{
Kinetics and mechanisms of stress relaxation in sputtered silver thin films
}

\author{
Quentin Hérault $^{\mathrm{a}, 1}$, Iryna Gozhyk ${ }^{\mathrm{a}}$, Matteo Balestrieri ${ }^{\mathrm{a}}$, Hervé Montigaud ${ }^{\mathrm{a}}$, \\ Sergey Grachev ${ }^{\mathrm{a}}$, Rémi Lazzari ${ }^{1, *}$ \\ ${ }^{a}$ Surface du Verre et Interfaces, UMR 125 CNRS/Saint-Gobain Recherche, 39 Quai Lucien \\ Lefranc BP 135, F-93303 Aubervilliers, France \\ ${ }^{b}$ CNRS, Sorbonne Université, Institut des NanoSciences de Paris, UMR 7588, 4 Place \\ Jussieu, F-75005 Paris, France
}

\begin{abstract}
The post-growth stress relaxation in thin polycrystalline Ag films, deposited by direct-current magnetron sputtering under different growth conditions, was explored through wafer curvature measurements. It exhibits exponential behaviour with three distinct characteristic time components $\tau$. The slowest one, namely $\tau_{t h} \simeq 200 \mathrm{~s}$, is ascribed to thermal stress inherent to the deposition method. In fact, the performed temperature measurements match perfectly with an exponential stress variation with heating or cooling, as predicted by a thermal exchange model detailed in this work. Based on a comparison between different deposition conditions (continuous/interrupted sputter deposition and evaporation), the case of Mo deposition and stress relaxation modelling, the remaining components are assigned to the out-diffusion of atoms from grain boundaries $\left(\tau_{g b} \simeq 3 \mathrm{~s}\right)$ and to changes in the grain surface shape induced by grain boundary grooving $\left(\tau_{\text {surf }} \simeq 20 \mathrm{~s}\right)$. The relaxation amplitude of the first mechanism varies linearly with the steady-state stress at the end of growth in agreement with theoretical expectations. Yet, that of the second one does not. However, clues point to a kinetic limitation of diffusion mechanism. This study provides proofs of the simultaneous occurrence of several mechanisms of stress relaxation in thin metallic films.
\end{abstract}

Keywords: polycrystalline films, stress, curvature measurements, relaxation, grain boundaries, grooving, silver, sputtering deposition

\footnotetext{
${ }^{*}$ Corresponding author

Email addresses: quentin.herault@saint-gobain.com (Quentin Hérault), iryna.gozhyk@saint-gobain.com (Iryna Gozhyk), matteo.balestrieri@saint-gobain.com (Matteo Balestrieri), herve.montigaud@saint-gobain.com (Hervé Montigaud), sergey.grachev@saint-gobain.com (Sergey Grachev), remi.lazzari@insp.jussieu.fr (Rémi Lazzari)
} 


\section{Introduction}

By leading to coating failures such as crack, peeling, delamination or blistering, the residual compressive or tensile stresses in polycrystalline thin films may be detrimental to the functionality and longevity of devices and in particular those exposed to harsh environments or to elevated temperatures [1]. Stress control in thin films requires a deep understanding of its physical origin(s). Kinetic-induced intrinsic stresses occurring during thin film growth result from a complex interplay between the film material, its thickness and the deposition technique and conditions. This is particularly relevant in the case of veryfar-from-equilibrium growth processes such as magnetron sputtering. In the Volmer-Weber growth mode of thin films, which is characteristic of the majority of polycristalline and even epitaxial metallic films on dielectrics, isolated islands nucleate and grow before impinging and coalescing. The percolation and formation of a continuous film follow. Each of these successive steps is assigned to a specific evolution of the parallel (or in-plane) stress state of the film [1-5]. For isolated islands, the observed compression is associated to the Laplace pressure [6]. From the start of coalescence up to the film percolation, the "zipping" of grain boundaries (GBs) [7, 8] generates a tensile stress, which is the result of a trade-off between the energies associated with the strain, the elimination of free surfaces and the creation of GB interfaces. After the formation of a continuous layer, the stress enters into a tensile (respectively compressive) state for low (high) mobility materials, referred to as type I (type II) materials [1, 5]. As in the structure zone model $[9,10]$, the homologous temperature, i.e. the ratio of growth $\left(T_{s}\right)$ to melting $\left(T_{m}\right)$ temperature, is often used as a criterion to distinguish between type I $\left(T_{s} / T_{m} \gtrsim 0.2\right)$ and type II $\left(T_{s} / T_{m} \lesssim 0.2\right)$ stress behaviour. Nevertheless, the characteristic diffusion length seems to be a better criterion, since it also accounts for the flux of incident atoms [11]. For type II materials, among which Ag studied herein is an archetype, the tensile stress associated to GB closure can actually switch to compressive stress upon increasing the thickness $[1,5]$. Conversely, if the growth is interrupted, an evolution towards tensile stress values is observed. But the puzzling fact is that if the growth is resumed, the stress returns back to the value achieved before interruption, as if the latter did not take place. The mechanism behind this reversible stress has been the object of a vivid debate in the literature [3, 12-18]. Several hypotheses have been put forward to explain this behaviour, all of them implying phenomena occurring in the region near film surface: (i) trapping and release of excess atoms between surface ledges [3], (ii) reversible change of surface stress due to the adatom population $[12,13]$ (iii) in- or out-diffusion of atoms from GBs thermodynamically dictated by the flux-driven difference of chemical potential between GB and free surface [14-16], (iv) reversible change of the surface morphology of individual grains $[17,18]$. A fruitful approach to the problem is to analyse the temporal evolution of the stress relaxation. Based on an accurate analysis of stress relaxation in Ag films with controlled grain size, Flötotto et al. pointed out the decisive role of the GB density (and a marginal role of the pre-interruption deposition rate) in the control of stress, thus ruling out the pre- 
vious interpretations (i) and (ii). Using a kinetic model based on atom insertion into GBs, Chason et al. predicted an exponential stress relaxation [14, 15] in apparent agreement with experiments in the case of Ag [14,19]. Through an exponential fit of the stress relaxation curve of evaporated $\mathrm{Ni}$ and Au films [20], Yu et al. evidenced a fast (characteristic time of $\sim 10^{2} \mathrm{~s}$ ) reversible and a slow $\left(\sim 10^{4} \mathrm{~s}\right)$ irreversible process, the latter being assigned to grain recrystallization in the bulk of the film based on microscopy analysis $[17,20]$. The slow stress relaxation process is the result of grain growth, which is driven by the reduction of interfacial energy and not really by strain minimization. Deepening of GB grooves by surface diffusion (which is the first step of dewetting [21]) was assumed to be at the origin of the fast release of a part of the compressive stress due to trapped atoms $[18,22,23]$. Upon growth resumption, the steady-state surface morphology with shallower grooves is recovered leading to the reversibility of the phenomenon.

Since relaxation depends not only on the material and the film microstructure, but also on the whole history of film growth (deposition technique and operating conditions), it is difficult to put forward a dominant mechanism by comparing one study to the other. In the present work, using the same deposition system, we show that both mechanisms involving (iii) the out-diffusion of atoms from GBs and (iv) the change of surface morphology are active in the release of compressive stress. To this end, stress relaxation dynamics has been analysed in continuous Ag films via multi-exponential fits. A special attention has been paid to the thermal stress (Section. 3.2) component by developing a heat transfer model that accounts for the specificity of sputtering deposition and explains the temperature evolution during deposition and relaxation (Section 3.3). Three exponential components (Section 3.1) have been identified during post-growth relaxation, one of which being related to thermal stress (Section. 3.4). The other two are ascribed to the combined out-diffusion of atoms from GBs and the change of the grain surface shape driven by GB grooving (Section 3.5). This assignment is based on a kinetic stress model (Section 3.6) and on a comparison with $\mathrm{Ag}$ evaporation (a slower deposition technique, free of energetics species) and also with Mo sputtering (the archetype of low diffusivity material with $T_{s} / T_{m}=0.10$ ). To this end, the versatility offered by the accessible sputtering deposition conditions (power/pressure) is used to induce changes in the final morphology and, therefore, to modulate the steady-state stress during growth and the relative amplitude and time constants of the three relaxation components. In addition, the interpretation is reinforced by a comparison between continuous growth and sequentially interrupted growth (Section 4) as well as by the impact of film thickness on stress relaxation (Section 5).

\section{Experimental}

Thin film growth was performed in a vacuum chamber with a base pressure of $10^{-4} \mu$ bar. Direct-current magnetron sputtering of $\mathrm{Ag}$ and Mo was achieved using $99.999 \%$ pure 2 inches targets set at $15 \mathrm{~cm}$ from the substrate. The surface 
of each target was cleaned by pre-sputtering before deposition. The Ar (purity $99.995 \%)$ sputtering gas pressure ranged from 2 to $50 \mu$ bar $(1-80 \mathrm{sccm})$. For $\mathrm{Ag}$, the power was set in a range between $50 \mathrm{~W}$ and $150 \mathrm{~W}$ for a cathode voltage of 345-440 V. The sample was left electrically floating and reached a potential of $\sim-7 \mathrm{~V}$. Within the power and gas pressure ranges explored in this work, the $\mathrm{Ag}$ deposition rate $R$ (Table 1), measured as explained in reference [24], increases with power $P$, but is nearly constant as a function of pressure $p$. The

\begin{tabular}{cccc}
$\begin{array}{c}\text { Power } \\
(\mathrm{W})\end{array}$ & $\begin{array}{c}\text { Pressure } \\
(\mu \text { bar })\end{array}$ & $\begin{array}{c}\text { Rate } \\
\left(\mathrm{nm}^{-1}\right)\end{array}$ & $\begin{array}{c}\text { Average kinetic energy } \\
\left(\mathrm{eV} \cdot \text { atom }^{-1}\right)\end{array}$ \\
\hline \hline 50 & 2 & 0.54 & 6.44 \\
75 & 2 & 0.72 & - \\
100 & 2 & 0.88 & - \\
125 & 2 & 1.09 & - \\
150 & 2 & 1.36 & - \\
50 & 3.5 & 0.52 & 3.38 \\
50 & 5 & 0.53 & 1.93 \\
50 & 10 & 0.54 & 0.49 \\
50 & 25 & 0.43 & $<0.04$ \\
50 & 50 & 0.43 & $<0.04$
\end{tabular}

Table 1: Measured $\mathrm{Ag}$ deposition rate $R$ and calculated average kinetic energy $E_{k}$ for the explored powers and pressures.

interrupted film growth was achieved with a pneumatic shutter placed in front of the target, allowing for controlled durations in opened/closed positions. As a basis for comparison in relaxation studies, a Mo film was grown at rate of $R=0.28 \mathrm{~nm} \cdot \mathrm{s}^{-1}(P=150 \mathrm{~W} ; p=2 \mu \mathrm{bar})$. Similarly, a Ag deposit was also obtained by evaporation at a much lower rate of $R=0.033 \mathrm{~nm} . \mathrm{s}^{-1}$. The used alumina crucible was heated at $1323 \mathrm{~K}$ and set at $15 \mathrm{~cm}$ from the substrate. $\mathrm{Si}(100)$ substrates (n or p-type doped; 2 inches) were used as supplied without removal of their native oxide (thickness $\sim 2 \mathrm{~nm}$ ), so that the initial growth takes place on a silica surface. The wafer thickness of $h_{s}=100$ and $150 \mu \mathrm{m}$ allowed reaching a good sensitivity in the curvature measurements used to obtain the stress evolution. Free bending of the wafer during film deposition was ensured simply by wafer laying onto a sample holder with three equally spaced tiny pins placed close to the edge. Just after deposition, some Ag films were covered in situ with a $3 \mathrm{~nm}$ thick $\mathrm{TiO}_{2-x}$ layer to prevent atmospheric effects during ex situ imaging. The film topography was then characterized in air by atomic force microscopy (AFM ICON from Bruker) running in tapping mode. The film thickness was determined by imaging a groove created on purpose in the layer [24].

In situ temperature measurements were performed with an alumina-shielded $\mathrm{K}$-type thermocouple (wire diameter $0.5 \mathrm{~mm}$ ) tightly fixed on the wafer surface with silver paint to ensure a good thermal contact. Data were collected using 
an electronic multimeter with a collection rate of one point per second, taking care there was no short circuit during deposition.

High-resolution stress measurements were performed with a home-made curvature monitoring setup based on digital-image correlation. In short, a random pattern is imaged by a camera equipped with a $100 \mathrm{~mm}$ objective lens, after its reflection on the substrate deposit face. Image correlation with respect to a reference image recorded before the growth or relaxation allows deducing sub-pixel displacements that can be linked to wafer curvature via a geometrical optics model. Precise calibration of the underlying experimental factors was obtained from the curvature measured ex situ with an optical profilometer on thick stable Mo films. The curvature $\kappa$ observed during growth or relaxation was then transformed into an average stress-thickness value $\bar{\sigma} h_{f}$ using the well-known Stoney equation [5, 25]

$$
\kappa=\frac{6 \bar{\sigma} h_{f}\left(1-\nu_{f}\right)}{E_{f} h_{s}^{2}},
$$

from the Young modulus $E_{f}$ and Poisson ratio $\nu_{f}$ of materials $\left(E_{A g}=83 \mathrm{GPa}\right.$; $\left.\nu_{A g}=0.37 ; E_{M o}=325 \mathrm{GPa} ; \nu_{M o}=0.3\right)[26]$.

Experiments were supplemented with simulations of transport of sputtered atoms from the target to substrate with the SIMTRA toolbox [27, 28] taking into account the specific configuration of our sputtering chamber [29]. The obtained average kinetic energy $E_{k}$ per $\mathrm{Ag}$ atom (Table 1 ) shows that a thermalization of atoms is theoretically achieved for deposition pressures above $p=25 \mu$ bar for which $E_{k}$ is below $0.04 \mathrm{eV}$.

\section{Mechanisms of stress relaxation after continuous deposition}

Fig. 1 depicts the stress-thickness evolution with film thickness during the growth of thin $\mathrm{Ag}$ film for various sputtering powers (Fig. 1-a) and gas pressures (Fig. 1-b). Fig. 2 shows the corresponding stress variation in $40 \mathrm{~nm}$ thick $\mathrm{Ag}$ films after deposition. Since $\mathrm{Ag}$ is a metal with a high homologous temperature $\left(T_{s} / T_{m}=0.24\right)$, the film stress follows a compressive-tensile-compressive scenario that is characteristic of a Volmer-Weber growth mode (Fig. 1). Above the percolation threshold, which is in the range of $\sim 10 \mathrm{~nm}$ herein, the stressthickness is characterized by a transition from a tensile peak to a compressive regime, the origin of which is related to GBs. The film stress enters a steadystate regime characterized by a stationary instantaneous stress. After growth, this compressive stress is gradually released towards tensile values as shown in Fig. 2 .

This section starts with the analysis of experimental data on stress relaxation via exponential fits (Section 3.1). Among the fitted components, the thermal stress (Section 3.2) is identified first (Section 3.4) through a comparison with 
temperature evolutions during and after the growth (Section 3.3). Then the remaining components are assigned to specific mechanisms (Section 3.5). Finally, a model of stress relaxation coupling atomic diffusion and grooving in GBs (Section 3.6) and its consequences (Section 3.7) is developed to support the previous identification. A thorough analysis of the role of the deposition parameters in the stress evolution during growth itself, from nucleation to percolation (Fig. 1), will be the topic of a separate article.
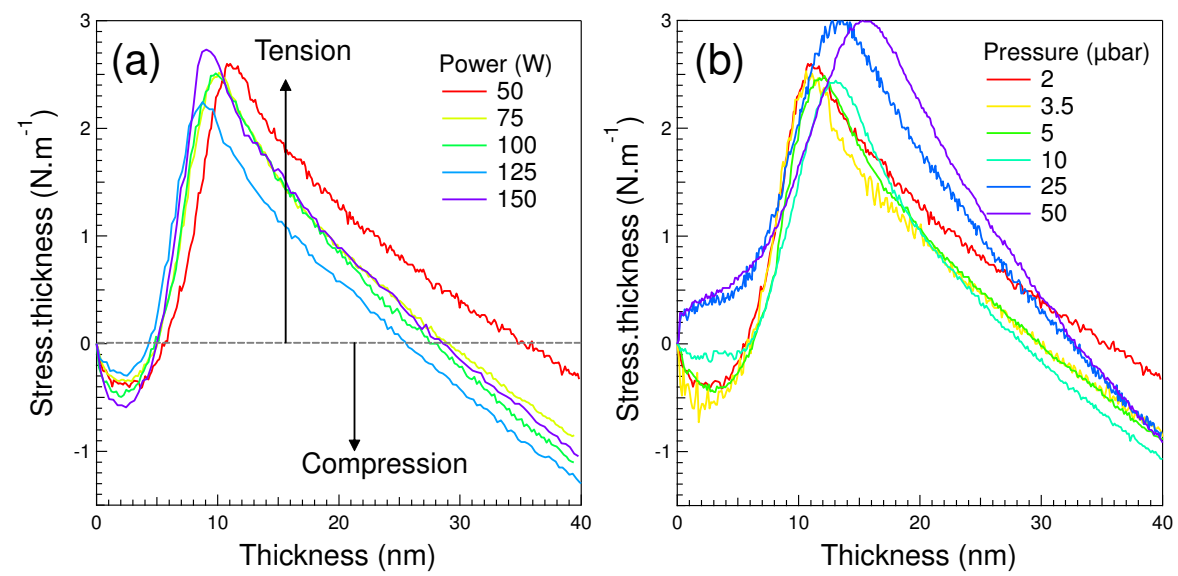

Figure 1: Stress-thickness evolution during continuous deposition of Ag at (a) fixed pressure $p=2 \mu$ bar for various powers and (b) at fixed power $P=50 \mathrm{~W}$ for various pressures.

\subsection{The three exponential components of stress relaxation}

Relaxation studies were performed during $600 \mathrm{~s}$ at a fixed film thickness $h_{f}=40 \mathrm{~nm}$ corresponding to a continuous and homogeneous layer according to in situ resistivity measurements [29]. If the plasma was switched off after the growth, Ar pressure was kept all along the relaxation process. The average stress variation $\Delta \bar{\sigma}(t)$ with respect to the value reached at the end of the growth is plotted in Fig. 2 as a function of power and pressure. Starting from a compressive stress value in the range of $\bar{\sigma}(t=0) \simeq-12$ to $-30 \mathrm{MPa}$, a rapid change of $\Delta \bar{\sigma}(t) \simeq 70-110 \mathrm{MPa}$ towards tension is systematically observed (Fig. 2). In magnetron sputtering such relaxation is not only due to intrinsic phenomena involving the film microstructure such as surface and grain boundaries, but also to the thermal stress related to the different film/substrate thermal expansion coefficients. Analogously to references [18, 20], the stress relaxation was fitted with the sum of three exponential terms, two of which are intrinsic to the film (indexed "surf" and "gb") and one related to the thermal stress (indexed "th"):

$$
\begin{aligned}
\Delta \bar{\sigma}(t) & =\Delta \bar{\sigma}_{t o t}-\Delta \bar{\sigma}_{\text {surf }} \exp \left(-t / \tau_{\text {surf }}\right) \\
& -\Delta \bar{\sigma}_{g b} \exp \left(-t / \tau_{g b}\right)-\Delta \bar{\sigma}_{t h} \exp \left(-t / \tau_{t h}\right) .
\end{aligned}
$$



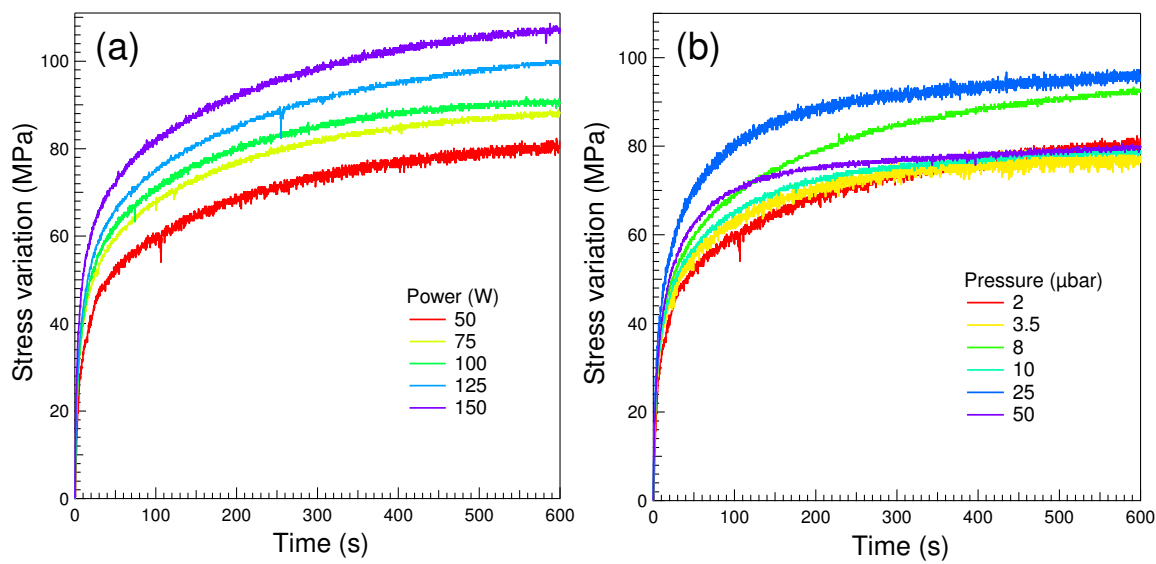

Figure 2: Post-growth stress variations $\Delta \bar{\sigma}(t)$ after deposition (a) at different powers and constant pressure $(p=2 \mu \mathrm{bar})$ and (b) vice-versa, different pressures and constant power $(P=50 \mathrm{~W})$.

Their origin will be discussed hereafter (Sections. 3.4-3.5). Fig. 3 depicts a fit of experimental data with one, two or three exponential terms. Beyond the suspected existence of these three terms [18, 20], a blind fit with only one or two components led to a worse agreement beyond the error bars. The evolution of fit parameters are presented in Fig. 4 as a function of power and pressure. At low deposition pressures $(p \simeq 2 \mu$ bar), the fit yields to three time constants with different orders of magnitude (Fig. 4-a,b): (i) $\tau_{\text {th }} \simeq 200 \mathrm{~s}$, (ii) $\tau_{\text {surf }} \simeq 20 \mathrm{~s}$ and (iii) $\tau_{g b} \simeq 3 \mathrm{~s}$. As seen in Fig. 4-a,b, the fitted time constants do not depend on power and only a weak dependence on pressure is observed for values below $10 \mu$ bar. By contrast, the corresponding amplitudes change with all the sputtering parameters, indicating that they might be dependent on the film microstructure and thermal history (Fig. 4-c,d).

In order to validate the existence of several exponential relaxation phenomena and to assign them, the present results are compared to those of: (i) a $40 \mathrm{~nm}$ film evaporated in high-vacuum $\left(R=0.033 \mathrm{~nm} \cdot \mathrm{s}^{-1}\right)$ and (i) a $91 \mathrm{~nm}$ Mo sputtered film $\left(R=0.28 \mathrm{~nm} . \mathrm{s}^{-1}\right)$ (Fig. S1 of supporting information). As expected for a poorly diffusive material $\left(T_{s} / T_{m}=0.1\right)$, the Mo film entered immediately into a tensile stress phase at the beginning of growth, and switched to a compressive phase only in the late stages of the growth due the well-known phenomenon of atomic peening [30, 31]. At the opposite, the evaporated Ag film followed a stress scenario similar to the sputtered Ag film. Yet, evaporation is free of energetic species and at such a low rate, the growth is closer to equilibrium. Both post-growth stress relaxations could be fitted conveniently with only two components (Fig. S1; Table 2). At last, the existence of two intrinsic relaxation times is further supported by the detailed stress relaxation study of Flötotto et al. [19] performed on the same $\mathrm{Ag} / \mathrm{SiO}_{2} / \mathrm{Si}$ system but grown by 


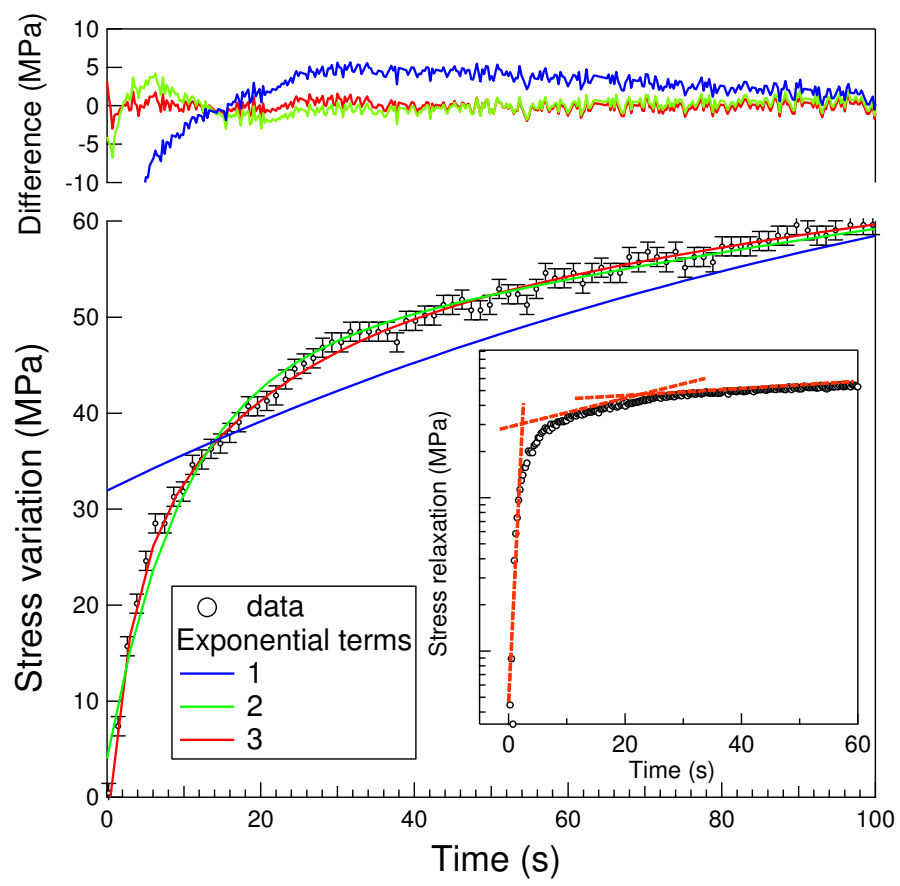

Figure 3: Example of fit of a stress relaxation curve (black points; $P=50 \mathrm{~W}$ and $p=2 \mu$ bar) with 1 (blue line), 2 (green line) and 3 (red line) exponential terms (see Eq. 2). Difference curves are plotted on the top scale. Data are plotted on a logarithmic scale in inset to show the three characteristic times. Fits are performed with a $\delta \bar{\sigma}= \pm 1 \mathrm{MPa}$ error bar. The data points removed for figure clarity are accounted for in fit. 

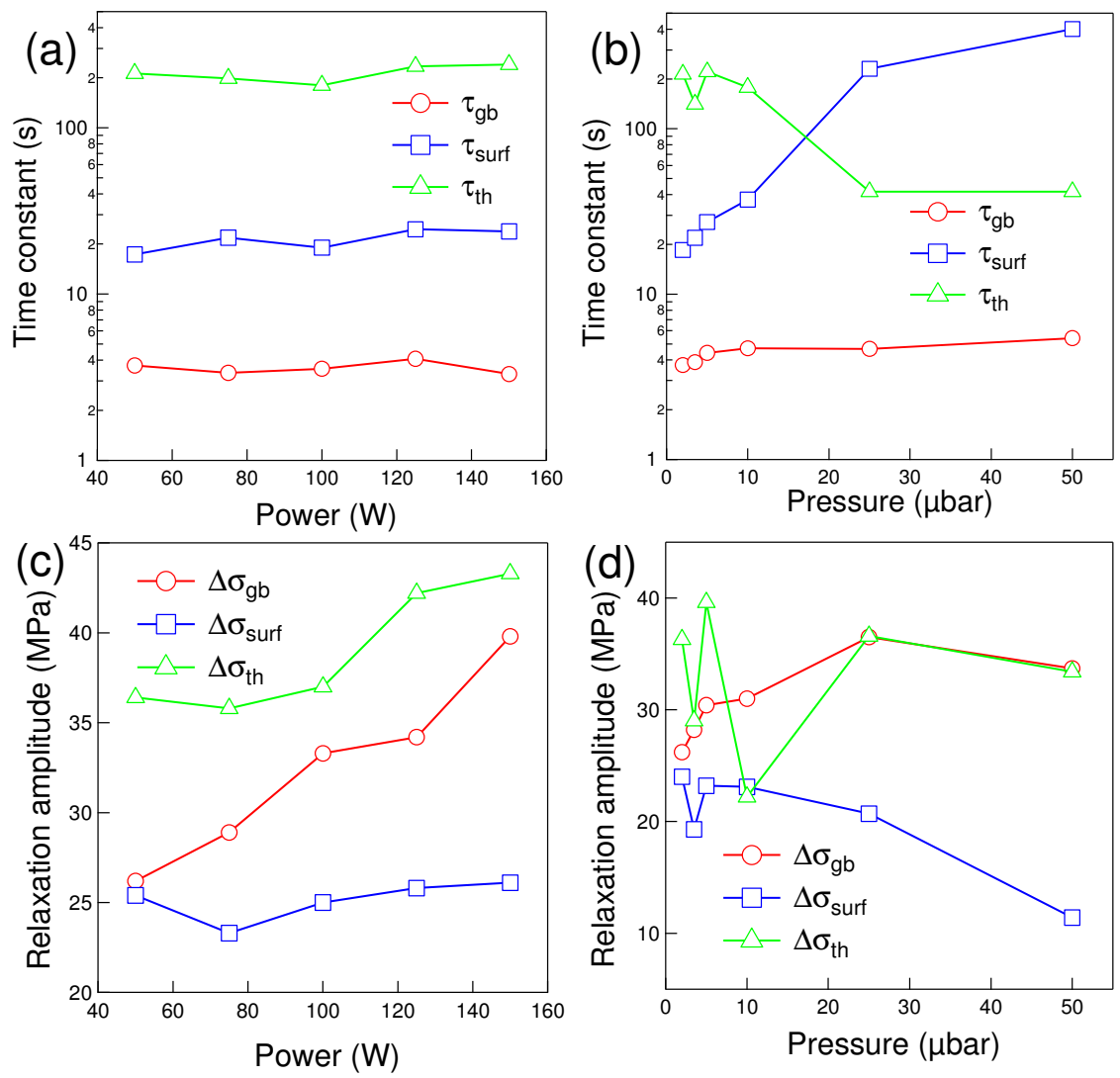

Figure 4: Exponential fitting parameters of the stress relaxation curves: (a)(b) time constants $\tau_{g b}, \tau_{\text {surf }}, \tau_{t h}$ and (c)(d) amplitudes $\Delta \bar{\sigma}_{g b}, \Delta \bar{\sigma}_{\text {surf }}, \Delta \bar{\sigma}_{t h}$ (see text for definitions; Eq. 2). Points correspond to growth at (a)(b) at fixed pressure $p=2 \mu$ bar or (c)(d) at fixed power $P=50 \mathrm{~W}$. The typical error bars are $\delta(\Delta \bar{\sigma})= \pm 1 \mathrm{Mpa}, \delta \tau_{g b}=0.3 \mathrm{~s}, \delta \tau_{\text {surf }}=2 \mathrm{~s}, \delta \tau_{\text {th }}=7 \mathrm{~s}$. 
vapour deposition; their thermal-stress free curves for similar growth rates show clearly two temporal regimes (see Fig. S2 of supporting information).

\begin{tabular}{ccccccc} 
System & $\begin{array}{c}\text { Thickness } \\
(\mathrm{nm})\end{array}$ & $\begin{array}{c}\Delta \bar{\sigma}_{g b} \\
(\mathrm{MPa})\end{array}$ & $\begin{array}{c}\tau_{g b} \\
(\mathrm{~s})\end{array}$ & $\begin{array}{c}\Delta \bar{\sigma}_{t h} \\
(\mathrm{MPa})\end{array}$ & $\begin{array}{c}\tau_{t h} \\
(\mathrm{~s})\end{array}$ & $\begin{array}{c}\Delta T_{t h} \\
(\mathrm{~K})\end{array}$ \\
\hline \hline Ag-evap. & 40 & 8 & 2.3 & 20.0 & 246 & -11.3 \\
Mo-sput. & 91 & 7.1 & 17.7 & 98.9 & 202.2 & -17.9
\end{tabular}

Table 2: Fit parameters of the stress relaxation curves (Fig. S1) for an evaporated Ag film $\left(p=10^{-4} \mu\right.$ bar; $\left.R=0.033 \mathrm{~nm} . \mathrm{s}^{-1}\right)$ and a $91 \mathrm{~nm}$ Mo film deposited by sputtering $(P=150 \mathrm{~W}$; $\left.p=2 \mu \mathrm{bar} ; R=0.28 \mathrm{~nm} . \mathrm{s}^{-1}\right)$. The temperature variation $\Delta T_{t h}$ is also given. See text and Eqs. 2,3 for definitions.

\subsection{The thermal stress component and the heat exchange model}

Assuming perfect adhesion at the film/substrate interface and mechanical equilibrium, any variation $\Delta T$ of temperature gives rise to a thermal stress [5, $32-35]$ that can be expressed as:

$$
\Delta \bar{\sigma}_{t h}=\frac{E_{f}}{1-\nu_{f}}\left(\alpha_{s}-\alpha_{f}\right) \Delta T,
$$

where $E_{f}$ is the Young modulus of the film material, $\nu_{f}$ its Poisson ratio and $\alpha_{f}, \alpha_{s}$ the linear thermal expansion coefficients of the film and the substrate, respectively. As sputtering is an energetic process, a temperature rise is to be expected during deposition and a cooling down during relaxation. To figure out the expected theoretical trend of temperature evolution in the film during deposition and its interruption, a heat exchange model was developed (see Section S1 for all details) including (i) the energy flux during deposition, (ii) the heat loss by thermal radiation and by conduction through the sample holder and the sputtering gas (both during deposition and relaxation) and (iii) the wafer thermal capacity. The main outcome is an exponential variation of temperature (Eqs. S10-S11):

$$
\begin{aligned}
\Delta T_{\text {heat }}(t) & =\Delta T_{\text {heat }}^{\text {max }}\left[1-\exp \left(-t / \tau_{\text {heat }}\right)\right] \\
\Delta T_{\text {cool }}(t) & =\Delta T_{\text {cool }}^{\text {max }} \exp \left(-t / \tau_{\text {cool }}\right),
\end{aligned}
$$

with a common time constant for heating (or deposition) and cooling (or relaxation), which is inversely proportional to the gas pressure. This predicted exponential behaviour of temperature is first confronted to direct measurements for various powers and pressures (Section 3.3) before identifying the thermal component of stress relaxation (Section 3.4).

\subsection{Temperature evolution during sputtering deposition and relaxation}

The substrate temperature was determined using a thermocouple glued onto the wafer surface during the deposition of a $40 \mathrm{~nm} \mathrm{Ag}$ film $(30-90 \mathrm{~s}$ of deposition; see Table 1), and during a fixed $600 \mathrm{~s}$ relaxation after the end of the 
growth (no plasma) but at a constant gas pressure. Fig. 5 depicts measured temperature variation during both growth and relaxation upon deposition with different magnetron powers (Fig. 5-a) or at various gas pressures (Fig. 5-b). During growth, a temperature rise of around $\Delta T_{\text {heat }} \simeq 10-20 \mathrm{~K}$ with respect to the original substrate/holder temperature $\left(T_{h}\right)$ is systematically observed (Fig. 5), with a slightly larger final temperature at higher powers and a lower one at larger pressures, as expected. No clear fingerprint of radiative loss changes due to variations of film emissivity around the percolation threshold $(\simeq 10 \mathrm{~nm})$ is found in the temperature profile (Fig. 5-a). This effect was already observed during the evaporation of $\mathrm{Ag}, \mathrm{Au}$ and $\mathrm{Cu}$, but at a much lower growth rate and for a larger emissivity contrast between deposited material and substrate (glass substrate $\left.\epsilon_{s}=0.92\right)[32,35]$.
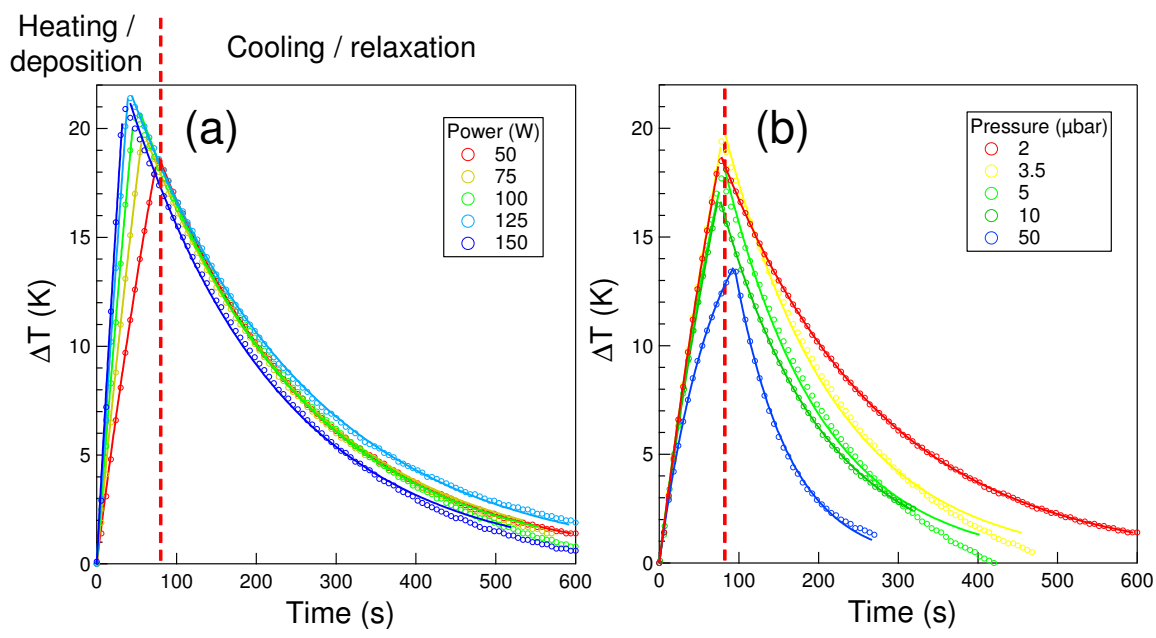

Figure 5: Temperature variation $\Delta T(t)=T_{s}(t)-T_{h}$ (circles: measurements; lines: fits) relative to the initial sample/holder temperature during sputtering deposition of a Ag film and during its cool-down under gas: (a) at different powers for a fixed pressure $p=2 \mu$ bar and (b) at different pressures for a fixed power $P=50 \mathrm{~W}$. Heating by deposition is performed until a common target thickness of $40 \mathrm{~nm}$ and cooling during a fixed duration of $600 \mathrm{~s}$ as used in stress relaxation measurements (Fig. 2).

Fig. 5 shows that both heating (deposition) and cooling (relaxation) can be conveniently fitted with exponential functions as theoretically predicted (Eqs. 4). However, for the highest deposition rates (at low pressures; Table 1), the short deposition time leads to a nearly linear increase of $T_{\text {heat }}(t)$ (Fig. 5), giving rise to strongly correlated $\Delta T_{\text {heat }}^{\text {max }}$ and $\tau_{\text {heat }}$ values in the fit. More reliable values can be obtained upon longer cooling (Fig. 5) or at larger pressures, for which the time constants are comparable with or smaller than the observation time. For this reason, at low pressures ( $p \leq 10 \mu \mathrm{bar}), \Delta T_{\text {heat }}^{\text {max }}$ was determined from the slope of the curve by setting $\tau_{\text {heat }}=\tau_{\text {cool }}$ as predicted by thermal modelling (Eq. S8). The obtained amplitudes $\Delta T_{\text {heat }}^{\text {max }}, \Delta T_{\text {cool }}^{\max }$ and time constants $\tau_{\text {heat }} \simeq \tau_{\text {cool }}$ are gathered in Fig. 6 as a function of power and pressure. 

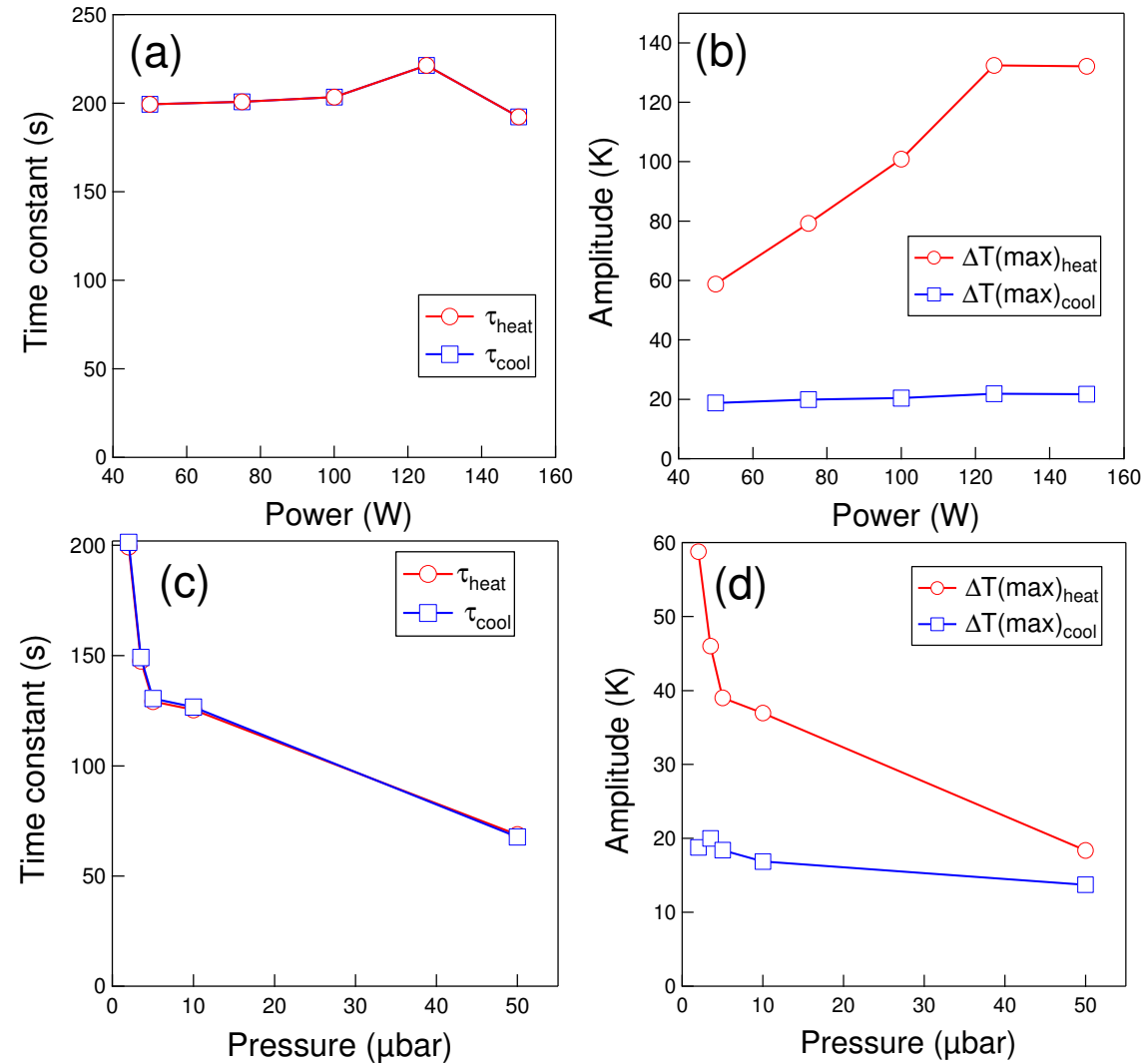

Figure 6: Exponential fitting parameters of the temperature evolution as a function of power (at constant pressure $p=2 \mu \mathrm{bar}$ ) and pressure (at constant power $P=50 \mathrm{~W}$ ): (a)(c) $\tau_{\text {heat }}$ and $\tau_{\text {cool }}$ time constants, (b)(d) amplitudes $\Delta T_{\text {heat }}^{\max }$ and $\Delta T_{\text {cool }}^{\max }$ (see text for definitions). The error bars are of the order of $\delta\left(\Delta T^{\max }\right) \simeq \pm 0.1 \mathrm{~K}$ and $\delta \tau \simeq \pm 2 \mathrm{~s}$ for an experimental uncertainty on temperature of $\delta T \simeq 0.5 \mathrm{~K}$. 
As expected from the heat transfer model, the deposition power does not impact the cooling relaxation time constant $\tau_{\text {cool }}$ (Fig. 6-a). At the same time, the characteristic time constants $\tau_{\text {heat }} \simeq \tau_{\text {cool }}$ (Fig. 6-c) are inversely proportional to the pressure, following the expectation of Eq. S8. The increase of the heating amplitude $\Delta T_{\text {heat }}^{\max }$ with power (Fig. 6-b) is compensated by a shorter deposition time (high deposition rate; Table 1), so that the temperature $T_{\text {end }}$ at the end of deposition is weakly dependent of power (Fig. 5). Consequently, the fitted $\Delta T_{\text {cool }}^{\max }=T_{\text {end }}-T_{h}$ values do not depend on the power either (Fig. 5-a). The asymptotic heating amplitude $\Delta T_{\text {heat }}^{\text {max }}$, and accordingly to Eq. S10 also the incoming heating flux $\Phi_{i n}$, increase with power (Fig. 6-b) and decrease with pressure (Fig. 6-d). Using the thermal capacity of the wafer $C_{s}=V C_{S i}$, with $V=0.3 \mathrm{~cm}^{3}$ its volume and $C_{S i}=0.7 \mathrm{~J} \cdot \mathrm{g}^{-1} \cdot \mathrm{K}^{-1}$ the specific heat of silicon [26], the amplitude of heating (Eq. S10) gives access directly to the flux of energy per unit of surface $\Phi_{i n} / A_{s}$. This value can be converted into the average deposited energy per atom $E_{a v}$ by normalizing to the atomic deposition rate $R_{a}=R \mathcal{N} \rho_{f} / M_{a}$ ( $R$ is the deposition rate, $\rho_{f}$ the atomic density, $M_{a}$ the molar mass and $\mathcal{N}$ the Avogadro's number). The values of $\Phi_{i n} / A_{s}$ $\left(\Phi_{i n} / A_{s}=7 \mathrm{~mW} . \mathrm{cm}^{-2}\right.$ at $P=50 \mathrm{~W}$ and $\left.p=2 \mu \mathrm{bar}\right)$ and $E_{a v}$ (Fig. 7 ) are in reasonable agreement with literature on sputtering deposition [36-41]. As demonstrated by the nearly constant $E_{a v}$ around 15 eV.atom ${ }^{-1}$ (Fig. 7), $\Phi_{\text {in }}$ scales linearly with the deposition rate $R$. Therefore, the thermalization of sputtered species with pressure predicted by SIMTRA simulation (Table 1) must be compensated by other heating channels to keep constant $E_{a v}$, as already demonstrated in the literature $[42,43]$.

\subsection{Identifying the thermal stress component}

For sputtered Ag film, a direct comparison of the order of magnitude of $\tau_{t h}$ (Fig. 4-a,b) with the cooling time constant $\tau_{\text {cool }}$ previously found by thermocouple measurements (Fig. 6-a,c) allows ascribing $\tau_{t h} / \Delta \bar{\sigma}_{t h}$ to the thermal stress at the film/substrate interface. This assignment can also be justified by comparing the expected temperature difference $\Delta T_{t h}$ at the end of deposition from the fitted amplitude $\Delta \bar{\sigma}_{t h}$ (Eq. $3 ; \alpha_{A g}=16.510^{-6} \mathrm{~K}^{-1} ; \alpha_{S i}=3.0810^{-6} \mathrm{~K}^{-1}$ ) to the measured value $\Delta T\left(t_{\text {end }}\right)$ (Fig. 5$)$. The obtained values around $20-25 \mathrm{~K}$ (see Table 3 ) are very close to those determined by thermocouple measurements (Figs. 5-6). Similarly, the changes of $\tau_{t h}$ and $\Delta \bar{\sigma}_{t h}$ with pressure (Fig. 4-b,d) follow those observed during cooling under different gas pressures (Fig. 6-c,d), both in terms of trend and of expected change of temperature, namely reaching values of the order of $\Delta T_{t h}=20 \mathrm{~K}$. Overall the thermal stress represents a sizable fraction of the total amplitude of stress relaxation (Fig. 2).

Thermal stress due to a temperature gradient through the wafer itself is ruled out because the predicted stress [32, 44]:

$$
\Delta \bar{\sigma}_{t h}^{s}=\frac{1}{6} \frac{E_{s}}{1-\nu_{s}} \alpha_{s} \Delta T_{s}
$$




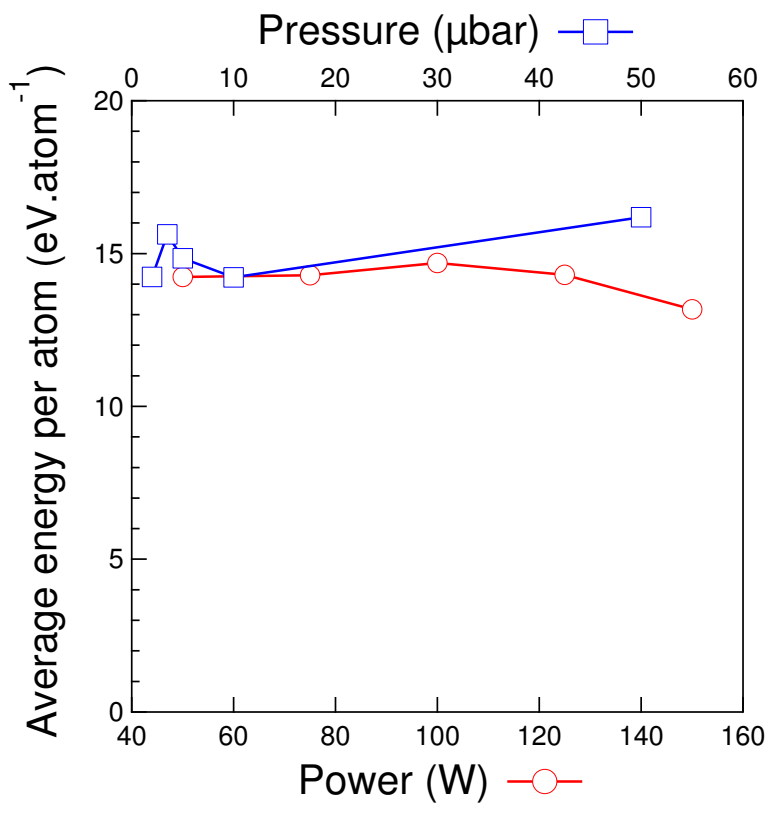

Figure 7: Average deposited energy per atom obtained from calorimetric equation (Eq. S10) and temperature measurements.

\begin{tabular}{cccc} 
Power $(\mathrm{W})$ & $\Delta \bar{\sigma}_{t h}(\mathrm{MPa})$ & $\Delta T_{t h}(\mathrm{~K})$ & $\Delta T_{t c}(\mathrm{~K})$ \\
\hline \hline 50 & 36.2 & -20.5 & -13.1 \\
75 & 35.7 & -20.3 & -15.1 \\
100 & 37.0 & -21.0 & -17.1 \\
125 & 42.0 & -23.8 & -16.6 \\
150 & 43.2 & -24.5 & -17.8
\end{tabular}

Table 3: Comparison for each deposition power between the expected temperature variation $\Delta T_{t h}$ corresponding to thermal stress amplitude and the value measured by thermocouple $\Delta T_{t c}$. 
amounts only to $0.1 \mathrm{MPa} . \mathrm{K}^{-1}\left(E_{S i}=113 \mathrm{GPa} ; \nu_{S i}=0.42\right)$ and would require a $\Delta T_{s}$ value of hundreds of kelvins to account for the observed relaxation amplitudes (Fig. 2). This is unlikely in the light of the present temperature measurements and of previous results [45]. Moreover, the incoming flux of energy estimated by calorimetry $\Phi_{i n} \simeq 10 \mathrm{~mW} \cdot \mathrm{cm}^{-2}$ is nearly entirely compensated by the thermal radiation escaping from the rear face $\Phi_{\text {rad }} \simeq 8 \mathrm{~mW} . \mathrm{cm}^{-2}$ $\left(\epsilon_{S i}=0.6>\epsilon_{A g}=0.02\right)$ for a difference of $20 \mathrm{~K}$ with the chamber walls (Eq. S3). This leads to a maximum temperature drop between the front and rear faces of $\Delta T_{r f} \simeq R_{t h} \Phi_{i n}=0.1 \mathrm{~K}$, accounting for the total thermal resistance of $R_{t h, s}\left(\mathrm{Ag} / \mathrm{SiO}_{2} / \mathrm{Si}\right) \simeq 1.10^{-2} \mathrm{~K} \cdot \mathrm{cm}^{2} \cdot \mathrm{W}^{-1}\left(R_{t h, s}=h / k\right.$ with $h$ the thickness and $k$ the thermal conductivity at $300 \mathrm{~K} ; k_{A g}=429 \mathrm{~W} \cdot \mathrm{m}^{-1} \cdot \mathrm{K}^{-1}$, $k_{\mathrm{SiO}_{2}}=1.4 \mathrm{~W} \cdot \mathrm{m}^{-1} \cdot \mathrm{K}^{-1}, k_{S i}=148 \mathrm{~W} \cdot \mathrm{m}^{-1} \cdot \mathrm{K}^{-1} ; h_{A g}=h_{f}=40 \mathrm{~nm}, h_{\mathrm{SiO}_{2}}=$ $\left.2 \mathrm{~nm} ; h_{S i}=150 \mu \mathrm{m}\right)$. In fact, if a thermal gradient exists in the substrate, it is in-plane and not across the wafer thickness because of the sample mounting on three feet. At last, some authors [46-49] claimed that a hot, but poorly conductive, "liquid-like" surface layer is induced by the arrival of metal atoms, leading to a surface temperature much higher than that of the substrate measured by the thermocouple. Upon cooling, such a gradient of temperature would give rise to a thermal stress as given by Eq. 5, but calculated with film parameters. Since the calculation for $\mathrm{Ag}$ gives $\Delta \bar{\sigma}_{t h}^{f} \simeq 0.36 \mathrm{MPa} \cdot \mathrm{K}^{-1}$, the assignment of any of the stress relaxation components to this phenomenon would require an unlikely temperature difference of several hundreds of kelvins. Such temperatures were indeed already pyrometrically measured in the case of $\mathrm{Ti}, \mathrm{Cu}$, and $\mathrm{Cr}$ sputtering $[48,49]$, but at a much higher incoming flux $\Phi_{\text {in }} / A_{s}$.

In the case of Mo sputtering, as $\tau_{t h} \simeq \tau_{\text {cool }}$, the slowest relaxation is obviously assigned to thermal stress (Table 2 ). Its amplitude suggests a cooling of $\Delta T_{t h}=-111 \mathrm{~K}$ with $\alpha_{M o}=5.010^{-6} K^{-1}$ (Eq. 3). Assuming $-\Delta T_{t h} \simeq$ $\Delta T_{\text {cool }}^{\text {max }}=\Delta T_{\text {heat }}^{\text {max }}\left[1-\exp \left(-t_{\text {dep }} / \tau_{\text {th }}\right)\right]$ (Eq. 2) where $\tau_{\text {dep }}=320 \mathrm{~s}$ is the deposition time, one finds a heating amplitude during deposition of $\Delta T_{\text {heat }}^{\text {max }}=140 \mathrm{~K}$ close to previous findings $\Delta T_{\text {heat }}^{\max }=130 \mathrm{~K}$ in the case of $\mathrm{Ag}$ at a similar power of $P=150 \mathrm{~W}$ (Fig. 6). While the corresponding flux of energy $\Phi_{i n} / A_{s}=$ $17 \mathrm{~mW} . \mathrm{cm}^{-2}$ (Eq. S10) is similar to that found for Ag, the average deposited energy per atom is much larger $E_{a v}=59.5 \mathrm{eV}$.atom ${ }^{-1}$ due to a lower Mo deposition rate. The lower sputtering yield of Mo compared to Ag implies not only a lower deposition rate, but also an enhancement of heating by Ar peening induced by ion neutralization and reflection at the target [36, 37]. Indeed, as SIMTRA simulations give an average kinetic energy similar to that of Ag for Mo $\left(E_{k}^{M o}=6.42 \mathrm{eV}\right.$ vs $E_{k}^{A g}=6.44 \mathrm{eV}$ at $\left.p=2 \mu \mathrm{bar}\right)$, and due to the small difference of their atomic mass values $\left(M_{a}^{A g}=96 ; M_{a}^{M o}=108\right)$ one would expect a similar thermal transfer from the impinging deposited atoms.

Finally, since the evaporator crucible was not thermally shielded during silver evaporation, a significant substrate heating occurred. Its time constant $\tau_{t h}=$ $246 \mathrm{~s}$ is larger than that observed for sputtering $\tau_{t h}=200 \mathrm{~s}$, a fact explained by the absence of a cooling mechanism by gas conduction (see Eq. S8). Owing to the 
long deposition time $\left(t_{d e p}=1200 \mathrm{~s}\right)$ compared to $\tau_{t h}=246 \mathrm{~s}$, Eqs. $4-3$ show that $\Delta T_{\text {heat }}^{\text {max }} \simeq-\Delta T_{t h}$. This corresponds to a heating flux of $\Phi_{\text {in }}=C_{s} \Delta T_{\text {heat }}^{\text {max }} / \tau_{\text {th }}=$ $22.5 \mathrm{~mW}$ (Eq. S9). The Stephan-Boltzmann equation (Eq. S1) tells us that the $1.5 \mathrm{~cm}$ nose of the alumina (emissivity $\epsilon=0.8$ ) crucible held at $1323 \mathrm{~K}$ emits $24 \mathrm{~W}$ in the half-space. The thermal radiation received by the substrate is proportional to its solid angle as seen from the evaporation cell (substrate area $A_{s}=20 \mathrm{~cm}^{2}$ at a distance $15 \mathrm{~cm}$ ), and amounts to $27 \mathrm{~mW}$. Since doped silicon is strongly absorbing at the crucible Wien wavelength $\left(\lambda_{w}=2 \mu \mathrm{m}\right.$ at $\left.1323 \mathrm{~K}\right)$, this value matches perfectly the above $\Phi_{i n}$ value estimated from thermal stress.

\subsection{Assignment of the other relaxation mechanisms}

Once the thermal stress component is identified and quantitatively explained, several reasonable hypotheses can be put forward to explain the presence of two additional intrinsic stress relaxation mechanisms for $\mathrm{Ag}$ sputtered films with time constants around $\tau_{g b} \simeq 3 \mathrm{~s}$ and $\tau_{\text {surf }} \simeq 20 \mathrm{~s}$ (Fig. 4): (i) bulk recrystallization $[17,20,50]$, (ii) out-diffusion of atoms from GBs promoted by the decrease of supersaturation at the film surface [14, 15, 51], (iii) healing of point defects created by atomic peening [52] or (iv) change of grain shape at the surface through GB grooving which is the first step of dewetting [18, 21-23].

Bulk recrystallization, i.e. grain growth, densifies the film by reducing the number of GBs; since the film adheres to the substrate, a tensile stress sets up. Already proposed in relaxation studies of evaporated Au or Ni films [20], bulk recrystallization is ruled out here on the basis of the much longer time scale of this process $\left(\sim 10^{4} \mathrm{~s}\right)$.

To disentangle the other mechanisms, the correlation of their relaxation amplitudes with the steady-state stress $\sigma_{s s}$ during the late stage of growth depicted by Fig. 8 turns out to be quite relevant. The amplitude of the fastest relaxation component $\left(\tau_{g b} \simeq 3 \mathrm{~s}\right)$ evolves linearly (slope $\left.\simeq 0.33\right)$ with $\sigma_{\text {ss }}$ while the slower one $\left(\tau_{\text {surf }} \simeq 20 \mathrm{~s}\right)$ is nearly constant (Fig. 8). The mechanism (ii) of atomic diffusion at GBs is known to scale with $\sigma_{s s}[14,15,51]$ (see below for details) thus supporting its assignment to $\tau_{g b} / \Delta \bar{\sigma}_{g b}$.

Let us consider other above-mentioned mechanisms. Contrarily to evaporation, peening in sputtering deposition $[5,30,31,53]$ leads to a compressive stress through the transfer of momentum from energetic particles (ions or backreflected neutrals) that favours implantation and/or creation of points defects leading to enhanced atom mobility and film densification. A relaxation towards less compressive/more tensile state (mechanism (iii)) is expected from the diffusion of bombardment-induced defects with a lower amplitude at larger growth pressures due to the thermalization of species but also a larger amplitude at larger power due to enhanced peening. Looking at Fig. 4, the component $\tau_{g b} / \Delta \bar{\sigma}_{g b}$ of stress relaxation clearly does not match these expected trends in particular with pressure at the opposite to $\tau_{\text {surf }} / \Delta \bar{\sigma}_{\text {surf }}$ (Fig. 4 ). But this latter does not show any clear correlation with the steady-state stress as one would 

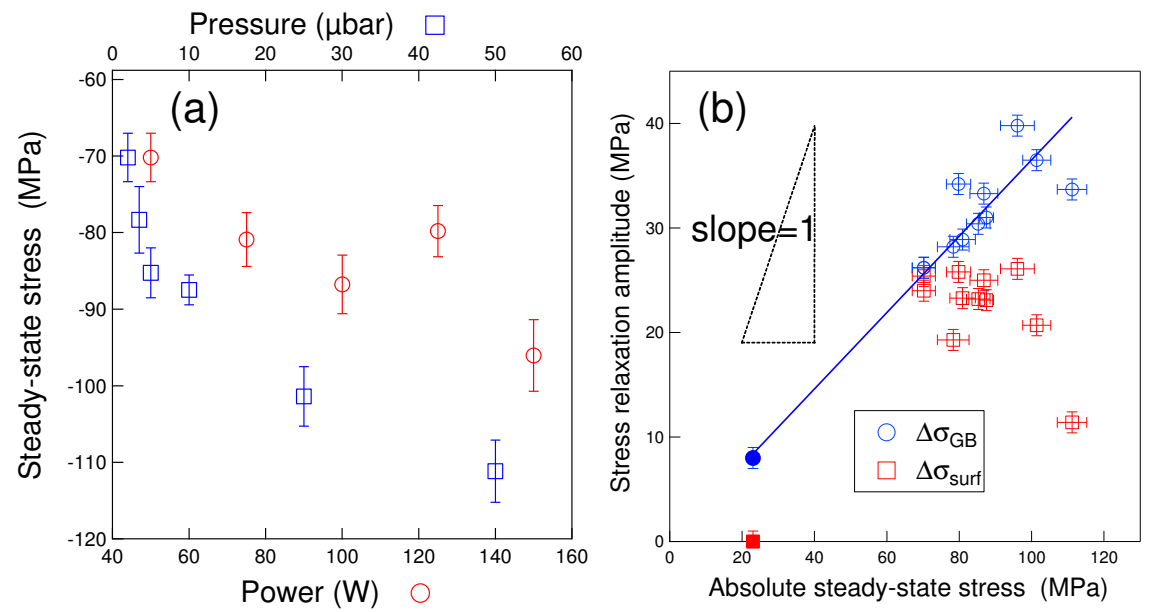

Figure 8: (a) Evolution of the compressive steady-state stress $\sigma_{s s}$ developed in the late stage of growth $\left(h_{f}=40 \mathrm{~nm}\right)$ for all the explored deposition conditions. (b) Correlation between the steady-state stress $\left|\sigma_{s s}\right|$ and the amplitudes of stress relaxation $\Delta \bar{\sigma}_{g b}, \Delta \bar{\sigma}_{\text {surf }}$. Filled points correspond to evaporation and the blue line to a linear regression calculated on the blue points. The unitary slope expected for unlimited diffusion at GBs is depicted by the dotted triangle.

suspect for peening $[5,53]$. Also, SIMTRA simulations [27, 28] performed for our sputtering geometry [29] (Table 1) predict a full thermalization of atoms above $p=25 \mu$ bar with an average kinetic energy of atoms below $0.04 \mathrm{eV}$, while sizeable $\Delta \bar{\sigma}_{\text {surf }} / \Delta \bar{\sigma}_{g b}$ amplitudes are still present. Finally, previous in situ photoemission analyses do not show the presence of implanted Ar [29] in the $\mathrm{Ag}$ films at the sensitivity limit of the technique ( $\sim 1 \%$.at). At last, one can point out that a stress relaxation line shape with two clear exponential components was already observed [14,19], although not discussed, in the case of $\mathrm{Ag}$ evaporated at rates similar to those used herein for sputtering, but of course in the absence of energetic species (see Fig. S2). In conclusion, the relaxation of peening-induced defects does not appear as dominant mechanism although it can not be fully ruled out at the lowest pressures; some effects on the compression peak in the pre-coalescence stage are visible (Fig. 1-b).

In coherence with previous observations for $\mathrm{Au}$ and $\mathrm{Ni}$ evaporated films [18, 20] and with theoretical predictions [54], the mechanism with the intermediate time constant $\left(\tau_{\text {surf }}\right)$ is rather assigned to GB grooving. This mechanism (iv) of stress relaxation driven by curvature-induced surface diffusion is quite relevant in the case of silver. This type II material with a sizeable surface diffusion coefficient is well-known to be prone to dewetting [21, 55-58]. Because of the out-of-equilibrium growth conditions, the dihedral angle of a GB groove is larger than the equilibrium one (fixed by the ratio between the GB energy and the grain surface energy). After growth, the groove deepens by surface diffusion 
towards its equilibrium angle with a speed that increases with the energy difference from equilibrium. This phenomenon is the first step of film dewetting, a process that ends up with the creation of holes in the film if the mobility is high enough [21, 58]. A less pronounced and slower relaxation at larger pressures is expected due to grains closer to their equilibrium shape as observed for $\tau_{\text {surf }} / \Delta \bar{\sigma}_{\text {surf }}$. As the opposite to sputtering, grains in the evaporated Ag film are expected to be closer to thermal equilibrium, because the process is slower and free of energetic species ( $E_{k}^{\text {evap }} \simeq 0.1 \mathrm{eV}$ for $\mathrm{Ag}$ atoms). A previous detailed analysis of the plasmonic response of islands during growth by sputtering and evaporation on a similar system in terms of wetting (Ag/alumina) [59] has shown that sputtering is characterized by a kinetic competition during coalescence between island lateral growth and return to equilibrium shape, leading to the formation of flatter particles and earlier percolation. In fact, percolation in evaporation is strongly delayed since coalescence is nearly self-similar in terms of aspect ratio with particle shape close to equilibrium [60-62]. Since the dihedral angle of GB grooves in evaporated films is closer to equilibrium, the component related to surface relaxation is not found in the relaxation data of evaporated samples (see Fig. S1-a and Table 2). The only remaining mechanism is the out-diffusion of atoms from GBs, the time constant of which is quite similar to that of sputtering $\left(\tau_{g b}^{\text {evap }}=2.3 \mathrm{~s}\right.$ vs $\left.\tau_{g b}^{\text {sput }}=3 \mathrm{~s}\right)$.

Being a metal with a high melting point, Mo poorly diffuses and the change of surface morphology through GBs grooving is therefore expected to be ineffective after the end of the growth, even at a final temperature of $\simeq 400 \mathrm{~K}$. Previous measurements $[5,53]$ have demonstrated that the steady-state stress in Mo films is inversely proportional to grain size thus highlighting the key role of GBs in stress build-up. The exact mechanisms, though, may be different from that of $\mathrm{Ag}$ for which the difference of chemical potential is the driving force of inwards/outwards diffusion of atoms during growth/relaxation. Defect creation/healing in the bulk of the grains [63], such as those arising from atomic peening $[30,31]$ can not be fully disregarded in the Mo case since the low sputtering yield of this material leads to strongly energetic back reflected Ar neutrals. But whatever the dominating mechanism [5] may be, the relaxation of the associated compressive stress is naturally assigned to the time constant $\tau_{g b}^{M o} \simeq 18$ s. If further experiments are required to understand the exact origin of stress relaxation in Mo films, this experiment with Mo confirms that the existence of two relaxation mechanisms in $\mathrm{Ag}$ is intrinsically related to its Volmer-Weber growth and to its large mobility.

The experimental evidences are in favour of an assignment of $\tau_{g b} / \Delta \bar{\sigma}_{g b}$ to out-diffusion of atoms from GBs and $\tau_{\text {surf }} / \Delta \bar{\sigma}_{\text {surf }}$ to GB grooving. Yet the interplay between them and the lack of correlation with $\sigma_{s s}$ of the latter (Fig. 8b) remains to be explained. The next section is dedicated to this point. 


\subsection{Coupling between atom diffusion at GBs and GB grooving in stress relax-} ation

Chason et al. suggested that the compression phenomena observed during growth of a continuous film is due to the reversible insertion of atoms within GBs $[14,15,51]$, driven by the difference of chemical potential between the surface and the interior of GBs due to supersaturation. By modelling the elastic deformation of atomic layers adjacent to a GB by a set of parallel linear springs, an exponential stress relaxation can be predicted [14, 15, 51] with an amplitude that scales linearly with the steady-state stress. The combined effect of GB grooving and atom diffusion along GBs on stress can still be accounted for within the same kinetic model by considering the regimes of high and low atomic diffusion along the GB. In the extreme case of high-GB mobility (i.e. when the rate of atom diffusion within the GB is much larger than the rate of transition between the surface and the GB), Chason et al. proposed a differential equation to describe the average stress $\bar{\sigma}[14,15,51]$ :

$$
\begin{aligned}
\frac{\partial \bar{\sigma}}{\partial t} & =\frac{\partial \bar{\Sigma}_{g b}}{\partial t}+\frac{\partial \bar{\Sigma}_{g r}}{\partial t} \\
\frac{\partial \bar{\Sigma}_{g b}}{\partial t} & =-\frac{a}{h \tau}\left[\bar{\sigma}-\sigma_{C}\right] \\
\frac{\partial \bar{\Sigma}_{g r}}{\partial t} & =-\frac{1}{h} \frac{\partial h}{\partial t}\left[\bar{\sigma}-\sigma_{T}\right] .
\end{aligned}
$$

In deriving Eq. 6, it is assumed that the depth $h$ of the GBs is equal to the film thickness $h_{f}$. During growth, the stress evolution results from the balance between the creation of compressive stress $\sigma_{C}<0$ due to atom insertion at GBs $\left(\frac{\partial \bar{\Sigma}_{g b}}{\partial t}\right.$ term in Eq. 6) and the tensile stress $\sigma_{T}>0$ due to GB zipping $\left(\frac{\partial \bar{\Sigma}_{g r}}{\partial t}\right.$ term in Eq. 6). The stress relaxation time:

$$
\tau=\frac{a L}{\beta D_{i}}
$$

scales with the lateral grain size $L$ and the effective diffusivity $D_{i}$ related to the hopping rate from the surface to the GB. $a^{3}$ is the atomic volume and $\beta$ a dimensionless parameter. In the steady-state regime observed in continuous film, the speed of creation of GB is constant and given by the growth rate (i.e. $\left.\frac{\partial h}{\partial t}=R\right)$; the instantaneous stress tends towards a compressive steady-state value $\sigma_{s s}<0$ as observed in Fig. 1. It can be obtained by solving Eq. 6:

$$
\sigma_{s s}=\frac{\sigma_{T}+\frac{a}{R \tau} \sigma_{C}}{1+\frac{a}{R \tau}}
$$

This theory explains satisfactorily the general dependence of $\sigma_{s s}$ on the growth parameters for many metals, and also accounts for the general trends observed during coalescence and even during grain coarsening in continuous films [14, 15, $50,51]$. Moreover Eq. 6 is not only valid during film growth $\left(\frac{\partial h}{\partial t}>0\right)$ but also during relaxation at constant thickness $\left(\frac{\partial h}{\partial t}=0\right)$ or if atomic diffusion allows GB 
grooving $\left(\frac{\partial h}{\partial t}<0\right)$. In the first case $\left(\frac{\partial h}{\partial t}=0\right.$, stress relaxes exponentially from its initial steady-state value $\sigma_{s s}$ to a final given value $\sigma_{C}$ with a time constant $h_{f} \tau / a$ :

$$
\bar{\sigma}_{g b}(t)=\sigma_{C}+\left(\sigma_{s s}-\sigma_{C}\right) \exp \left(-\frac{a t}{h_{f} \tau}\right)
$$

Distinguishing between incoming and outgoing rates of atoms at GBs leads to a refined version of stress relaxation dynamics [64] that poorly differs from the exponential case Eq. 9 in the regime of small stress relaxation (see Fig. S3). Assuming now an exponential GB grooving of amplitude $\Delta h_{f}$ and time constant $\tau_{g v}$ :

$$
h=h_{f}-\Delta h_{f}\left[1-\exp \left(-t / \tau_{g v}\right)\right],
$$

Eq. 6 can be solved by finite difference method as shown in Fig. 9. It is quite
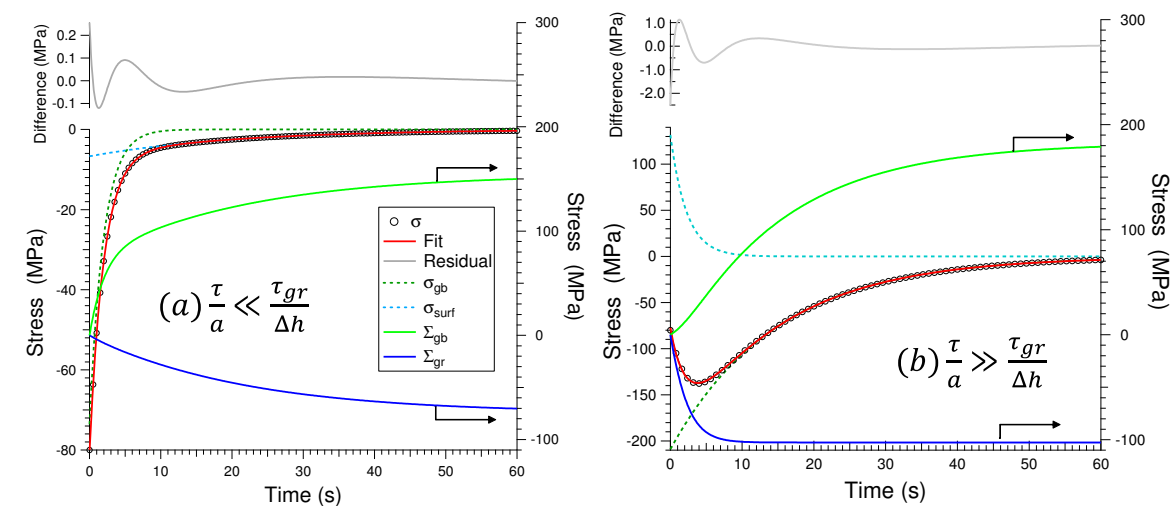

Figure 9: Finite difference solution (circles) of stress evolution (Eq. 6) for exponential GB grooving (Eq. 10) in the two limit cases: (a) $\frac{\tau}{a} \ll \frac{\tau_{g v}}{h_{f}}\left(\tau=2 \frac{a}{h_{f}} \mathrm{~s} ; \tau_{g v}=20 \mathrm{~s}\right)$ and (b) $\frac{\tau}{a} \gg \frac{\tau_{g v}}{h_{f}}\left(\tau=20 \frac{a}{h_{f}} \mathrm{~s} ; \tau_{g v}=2 \mathrm{~s}\right)$. The red line corresponds to the fit of the calculated stress relaxation (circles) with two exponential terms $\bar{\sigma}_{g b}$ and $\bar{\sigma}_{\text {surf }}$ (dotted lines). The difference is displayed on the top scale. The tensile relaxation $\bar{\Sigma}_{g b}$ due to out-diffusion of atoms from GBs (green line) and the compressive relaxation due to grooving $\bar{\Sigma}_{g r}$ (blue line) are displayed on the right scale. The numerical parameters are: $a=0.257 \mathrm{~nm}, h_{f}=40 \mathrm{~nm}, \Delta h_{f}=10 \mathrm{~nm}$, $\sigma_{C}=0 \mathrm{MPa}, \sigma_{T}=250 \mathrm{MPa}, \sigma_{s s}=-80 \mathrm{MPa}$. The chosen $\sigma_{T}$ and $\sigma_{s s}$ values correspond to the average stress at tensile peak and to the steady-state value observed during Ag growth (Fig. 1). See text for parameter definitions.

instructive to analyze the evolution of stress with time in two limit cases of fast (Fig. 9-a; $\tau / a \ll \tau_{g v} / \Delta h_{f}$ ) and slow (Fig. 9-b; $\tau / a \gg \tau_{g v} / \Delta h_{f}$ ) GB grooving kinetic compared to out-diffusion of atoms from GB. As in the case of growth, $\bar{\Sigma}_{g b}$ and $\bar{\Sigma}_{g r}$ compete to lead to a complex trend for $\bar{\sigma}$. Besides the direct fit of simulations (dotted lines in Fig. 9), an analytic analysis of Eqs. 6 detailed in Section S3 of supporting information shows that, for very distinct time constants $\tau_{g v}$ and $h_{f} \tau / a$, the total stress change can be decomposed into two exponential components, just like it was done in the analysis of the experimental data (Eq. 2 and Fig. 3) even if the evolutions of the counteracting tensile $\bar{\Sigma}_{g r}$ and 
compressive $\bar{\Sigma}_{g b}$ components themselves do not follow this exponential trend. In the case $\tau / a \ll \tau_{g v} / \Delta h_{f}$ and $\Delta h_{f} \ll h_{f}$ (Section S3), the amplitudes of these exponential components are given by:

$$
\begin{aligned}
\Delta \bar{\sigma}_{g b} & \simeq \sigma_{C}-\frac{\tau}{\tau_{g v}} \frac{\Delta h}{a}-\sigma_{s s} \\
\Delta \bar{\sigma}_{\text {surf }} & \simeq \frac{\tau}{\tau_{g v}} \frac{\Delta h}{a} .
\end{aligned}
$$

The distinct experimental dependence of $\Delta \bar{\sigma}_{\text {surf }}$ and $\Delta \bar{\sigma}_{g b}$ with respect to $\sigma_{s s}$ (Fig. 8) and the overall stress relaxation line shape with $\tau_{g b} \ll \tau_{\text {surf }}$ (Fig. 9-a versus Fig. 2) further reinforce the proposed identification: $\tau_{g b}=\tau h_{f} / a$ and $\tau_{\text {surf }}=\tau_{g v}$.

\subsection{Stress gradient along GBs and GB grooving dynamics}

Let's come back to the found proportionality factor between $\Delta \bar{\sigma}_{g b}$ and $\sigma_{s s}$ (Fig. 8-b) that does not match theoretical expectation of Eq. 11. It is worth noticing that the experimental values of $\Delta \bar{\sigma}_{g b}$ and $\Delta \bar{\sigma}_{\text {surf }}$ are of the same order of magnitude (Fig. 4-c,d). Meanwhile theory predicts a much more modest value for $\Delta \bar{\sigma}_{\text {surf }}$ (Eq. 12; see Fig. 9). Moreover, the nearly constant $\tau_{g b}$ (Fig. 4a,b) observed even in cases of evaporation (Table 2) and interrupted growth (see Section 4) contradicts the dependence of $\tau$ on grain size (Eq. 7), which is known to change with growth conditions [19, 29, 65]. The obtained experimental values of the steady-state stress $\sigma_{s s}$ and fitted relaxation times $\tau_{g b}$ follow the predicted trend for fast atomic diffusion (Eq. 8) but leads to unphysical stress values (see Section S4 and Fig. S4). Finally, only a fraction of $\sigma_{s s}$ is actually released during relaxation (see Fig. 8-b) as if a kinetic limitation was involved. As already suggested by other authors [14, 51, 66], these discrepancies might originate from a non-homogeneous stress profile across the film/GB thickness [66] due to a limited diffusion along GB. Guduru et al. [66] generalized the initial idea of Chason by coupling the kinetic equation of atom incorporation into the GB $[14,15,51]$ to an atomic stress diffusion equation. The driving force for the atomic diffusion is the gradient of chemical potential induced by the stress in the GB as in Cooble creep mechanism. The model is not analytical and requires a numerical solution, but some general considerations can be made. The build-up of the stress gradient over the layer thickness during growth depends on two time scales: (i) that of the diffusion of stress along the GBs $t_{d}=\frac{h_{0}^{2} L k T}{a^{3} \delta D_{g b} M_{f}}$ and (ii) the total time required to deposit the layer $t_{g}=\frac{h_{0}}{R}$. $h_{0}$ corresponds to the onset of the validity of the model, i.e. the formation of a continuous layer. It will be assumed to be $h_{0} \simeq 10 \mathrm{~nm}$ hereafter (see Fig. 1). $D_{g b}$ is the diffusion coefficient along a GB of width $\delta$. The product $\delta \times \delta D_{g b}$ is the quantity typically determined in the so-called type-B kinetic measurement of the diffusion of radiotracer elements involving both diffusion along the GB and into adjacent crystals. At low temperatures, only the former mechanism is active (so called type C-kinetic). Sommers et al. [67] accurately measured both 
in the case of $\mathrm{Ag}$ and the values extrapolated for the growth temperature of this work $(\sim 320 \mathrm{~K})$ are $\delta . D_{g b} \simeq 1.410^{-29} \mathrm{~m}^{3} \cdot \mathrm{s}^{-1}$ and $D_{g b} \simeq 1.110^{-17} \mathrm{~m}^{2} \cdot \mathrm{s}^{-1}$. With $L \simeq h_{f}=40 \mathrm{~nm}[29], a^{3}=17.1 \AA^{3}, M_{f}=E_{f} / 1-\nu_{f}=131.7 \mathrm{GPa}$ and $R=0.54 \mathrm{~nm} . \mathrm{s}^{-1}$ (Table 1 ), one finds $t_{d} \simeq 535 \mathrm{~s}$ and $t_{g} \simeq 18.5 \mathrm{~s}$. Using these time values, Guduru et al. [66] defined a dimensionless growth rate $\tilde{R}=\frac{4 \pi l_{0} t_{d}}{L t_{g}}$ to distinguish between fast and slow stress diffusion cases. The herein obtained value $\tilde{R} \simeq 100 \gg 1$ in the case of sputtering demonstrates that the film deposition rate is fast compared to the stress diffusion time through the film thickness, resulting in sizeable stress gradient along the film thickness.

At the extreme limit of very low-GB mobility [15] (without grooving), only the stress in the last atomic plane can relax via the out-diffusion of atoms, leading to an average stress that reads:

$$
\bar{\sigma}_{g b}(t)=\frac{a}{h_{f}}\left[\sigma_{C}+\left(\sigma_{s s}-\sigma_{C}\right) \exp \left(-\frac{t}{\tau}\right)\right] .
$$

Again, the stress $\bar{\sigma}_{g b}(t)$ relaxes exponentially, but it is characterized by a much lower amplitude and a faster characteristic time compared to the opposite case of fast diffusion limit (Eq. 9; $\frac{a}{h_{f}}=0.006$ ). Therefore, in the spirit of Eqs. 9 and 13 , a natural ansatz in the case of intermediate diffusion rates would read:

$$
\bar{\sigma}_{g b}(t) \simeq \frac{h_{g b}}{h_{f}}\left[\sigma_{C}+\left(\sigma_{s s}-\sigma_{C}\right) \exp \left(-\frac{a t}{h_{g b} \tau}\right)\right]
$$

where $h_{g b}$ is a measure of the stress gradient depth. In other words, it is equivalent to say that the release of stored stress $\sigma_{s s}-\sigma_{C}$ happens over a limited depth, i.e. $h_{f} \Delta \bar{\sigma}_{g b}=h_{g b}\left(\sigma_{C}-\sigma_{s s}\right)$. The linear regression of Fig. 8-a gives $h_{g b} \simeq 14 \mathrm{~nm}$ and $\sigma_{C} \simeq 0 \mathrm{MPa}$. Actually, $h_{g b}$ is very close to the diffusion length $L_{g b}=\sqrt{3 \tau_{g b} D_{g b}} \simeq 10 \mathrm{~nm}$ calculated for $3 \tau_{g b}$, the time to release exponentially most of the GB stress amplitude by atomic diffusion. Finally, the small $\sigma_{C}$ corresponds to an equilibrium of chemical potentials between GBs and surface $[14,16]$.

As the experimental relaxation times are well decorrelated $\left(\tau_{s u r f} / \tau_{g b} \gtrsim 6\right)$, the groove deepening does not relax the compressively stressed material inside the GBs, as initially supposed [18]. It most likely gives access to deeper stressed layers, so that the atom out-diffusion mechanism that already emptied surface layers can occur. A rough estimate of the groove deepening $\Delta h_{f}$ is obtained by assuming that the observed amplitude $h_{f} \Delta \bar{\sigma}_{\text {surf }}$ is the sum of the relaxation $\sigma_{s s}-\sigma_{C}$ over a depth $\Delta h_{f}$ of buried layers and of a release of residual stress $\sigma_{C}$ due to GB opening over the depth $\Delta h_{f}$ :

$$
h_{f} \Delta \bar{\sigma}_{\text {surf }} \simeq \Delta h_{f}\left(\sigma_{C}-\sigma_{s s}\right)+\Delta h_{f} \sigma_{C}=-\Delta h_{f} \sigma_{s s}
$$

Calculations give again a value of $\Delta h_{f} \simeq 10 \mathrm{~nm}$ (see Fig. 10). As described in the seminal work of Mullins [68], where the diffusive mass transport is driven from the local curvature at the surface, a GB groove deepens as:

$$
\Delta h_{g r}=0.973 \tan \xi(B t)^{1 / 4}
$$




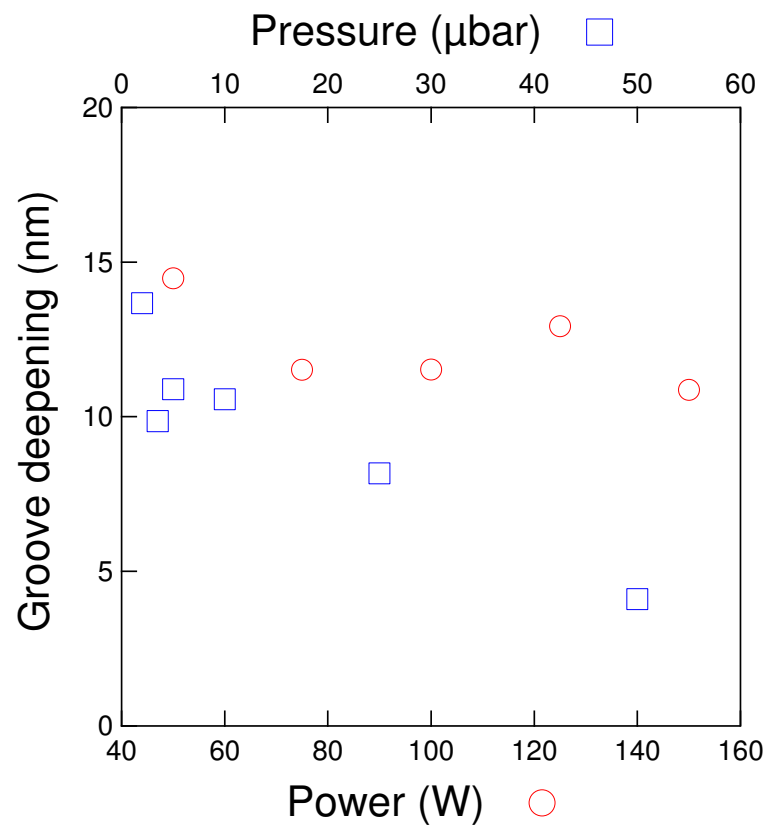

Figure 10: Evolution of the estimated groove deepening $\Delta h_{f}$ during relaxation, as a function of the sputtering power and pressure.

where $\xi$ is the initial opening angle of the groove and $B=\frac{D_{s} \gamma a^{3} \rho}{k T}$ a proportionality factor calculated from the surface diffusion coefficient $D_{s}$, the surface energy $\gamma$ and the surface density of atoms $\rho$. Using $T=320 \mathrm{~K}, D_{s}=2.510^{-12} \mathrm{~cm}^{2} . \mathrm{s}^{-1}$, $\gamma=1.2 \mathrm{~J} \cdot \mathrm{m}^{-2}$ (References $[69,70]$ ) and $\tan \xi \simeq 1$, one finds that the groove deepening reaches a depth of $\Delta h_{g r} \simeq 7 \mathrm{~nm}$ after $\sim 3 \tau_{\text {surf }}$, that corresponds to the time to relax most of $\Delta \bar{\sigma}_{\text {surf }}$. The apparent good agreement with the above estimate of $\Delta h_{f}$ (Fig. 10) hides somehow the fact that the capillar approach of a curvature-driven diffusion ignores the crystalline nature of the grains [58, 59]. In principle, a groove will lock when it reaches its equilibrium angle, in similar way as observed during film dewetting [58].

Nevertheless, the $\Delta h_{f}$ variation with power and pressure parallels the thickness evolution of the tensile peak and therefore the percolation threshold (Fig. 1). This correlation is ascribed to the presence of grains, and therefore to GBs, closer to the equilibrium shape at lower pressures in agreement with the reduced deposition rate and the thermalization of the atomic species (Table 1). The slowdown of the groove deepening at higher pressures ${ }^{1}$ (Fig. 4-d) reinforces

\footnotetext{
${ }^{1} \mathrm{~A}$ variation of the kinetic of diffusion due to a faster cooling at lower pressures can not explain a change of one order of magnitude of $\tau_{\text {surf }}$. Taking a constant diffusion length scale $\sqrt{D \tau_{\text {surf }}}$, an Arrhenius dependence of the surface diffusion coefficient yields to $\frac{\Delta \tau_{\text {surf }}}{\tau_{\text {surf }}}=$
} 
this interpretation.

\section{Stress relaxation after interrupted deposition}

Intermediate relaxations can be used as a lever to control the film microstructure and to confirm the previous interpretations obtained in the case of continuous sputter deposition. The idea is to interrupt the growth periodically with a shutter to allow for intermediate relaxation periods while recording stress-thickness during the whole process. Interruptions favour the return to thermodynamic equilibrium, the change of grain shape at the film surface through diffusion and the heat dissipation thus resulting in lower mechanical post-deposition relaxation amplitudes. Keeping in mind the orders of magnitude of the relaxation time constants $\left(\tau_{g b} \simeq 3 \mathrm{~s}, \tau_{\text {surf }} \simeq 20 \mathrm{~s}\right.$ and $\left.\tau_{\text {th }} \simeq 200 \mathrm{~s}\right)$, two ratios $\Delta t_{o n} / \Delta t_{\text {off }}$ of growth/interruption times were selected at $1 \mathrm{~s} / 5 \mathrm{~s}$ and $1 \mathrm{~s} / 15 \mathrm{~s}$ to favour, or not, the change of grain surface shape. Continuous and sequential growths were compared for similar sputtering conditions $(P=50 \mathrm{~W}$; $p=5 \mu$ bar). The number of periods $n$ was adapted to reach the same final thickness $h_{f}=40 \mathrm{~nm}$.

Figs. 11-a,b show the stress-thickness evolution during continuous and interrupted growth and its post-deposition relaxation. It is interesting to note that the tensile peak and the percolation threshold are shifted to higher thickness upon increasing the duration of deposition interruption. During interruptions, the isolated islands tend to dewet, thus delaying the formation of GBs. Once GBs are formed, GB grooving takes place during the relaxation intervals. This phenomenon leads to the formation of larger grains as shown by AFM images of the deposited films at the end of the growth (Fig. 12).

The previously introduced fit with three exponential terms (Eq. 2) was applied to the final relaxation at the end of growth of the $40 \mathrm{~nm}$ film (Fig. 11-c,d). The thermal stress component could again be easily identified thanks to its specific time constant. Similarly to the continuous process, the temperature at the end of deposition $\Delta T_{t h}$ can also be calculated from the thermal stress relaxation amplitude (Eq. 3), and compared to the final estimated temperature increase $\Delta T_{\text {end }}^{n}$ after $n=74$ cycles of interrupted growth. By neglecting transient effects, this latter can be obtained from a straightforward geometric series sum applied to the continuous heating/cooling temperature dynamics (Eqs. 4):

$$
\begin{aligned}
\Delta T_{\text {end }}^{n} & =\Delta T_{\text {heat }}^{\text {max }} \exp \left(-\Delta t_{\text {off }} / \tau_{\text {cool }}\right) \\
& \times\left[1-\exp \left(-\Delta t_{\text {on }} / \tau_{\text {heat }}\right)\right] \frac{1-\exp \left(-n \Delta t_{\text {on }} / \tau_{\text {heat }}\right)}{1-\exp \left(-\Delta t_{\text {on }} / \tau_{\text {heat }}\right)} .
\end{aligned}
$$

\footnotetext{
$-\frac{\Delta D}{D}=-\frac{E_{a} \Delta T}{k T^{2}}$. For a $\Delta T \simeq-20 \mathrm{~K}$ and wide range of activation energy $E_{a}=0.1-0.5 \mathrm{eV}$, the variation of the relaxation time remains modest in the range of 0.3-1.5 and can not explain the experimental observations (Fig. 4-b).
} 

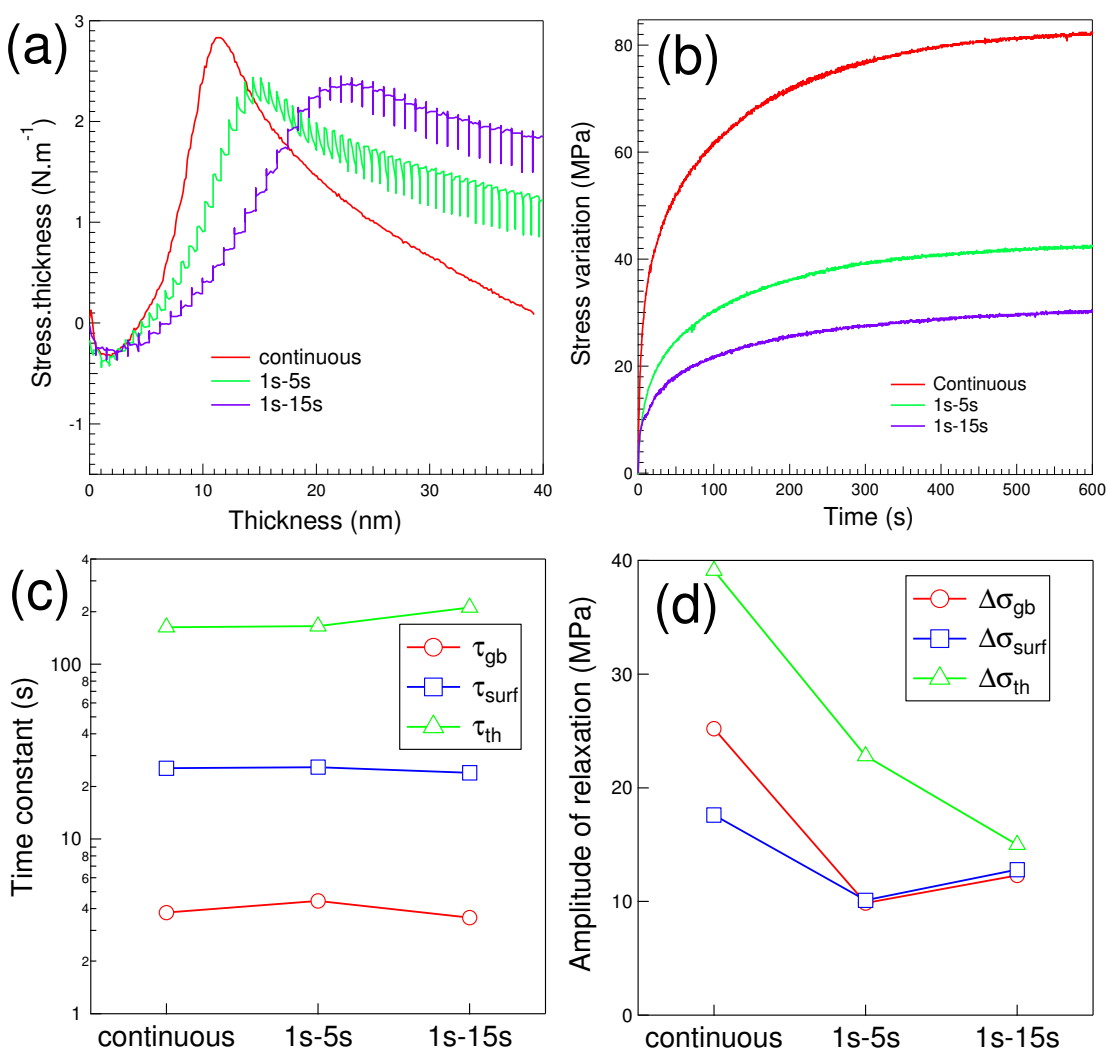

Figure 11: (a) Stress-thickness evolution during continuous or interrupted sputter depositions of $\mathrm{Ag}$ as indicated in figure $(P=50 \mathrm{~W} ; p=5 \mu \mathrm{bar})$. Relaxation during growth interruptions gives rise to jumps since data are plotted as a function of thickness. (b) Stress relaxation versus time at the end of growth. Results of exponential fitting of figure b : (c) time constants and (d) amplitudes for different ratio $\Delta t_{o n} / \Delta t_{\text {off }}$.

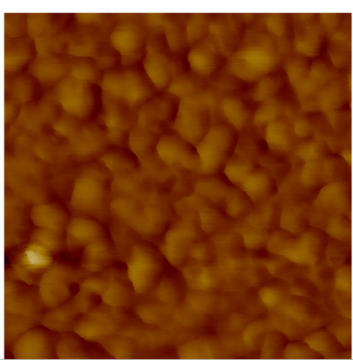

(a) Continuous

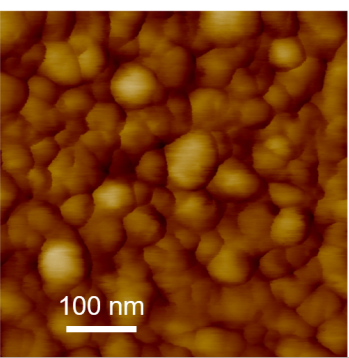

(b) $1 \mathrm{~s}-5 \mathrm{~s}$

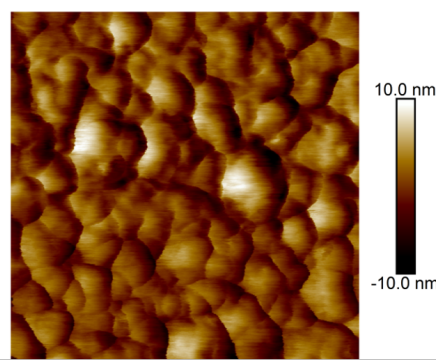

(c) $1 \mathrm{~s}-15 \mathrm{~s}$

Figure 12: Topographic AFM images obtained after (a) continuous and sequential growths at (b) $\Delta t_{o n}=1 \mathrm{~s} / \Delta t_{o f f}=5 \mathrm{~s}$ and (c) $\Delta t_{o n}=1 \mathrm{~s} / \Delta t_{o f f}=15 \mathrm{~s}$. Silver layers were capped by a thin $\mathrm{TiO}_{2-x}(3 \mathrm{~nm})$ layer to prevent atmospheric effects. 
In a reassuring way, both temperature increases $\Delta T_{t h}$ and $\Delta T_{e n d}^{n}$ are in close agreement (Table 4).

\begin{tabular}{cccc}
$\frac{\Delta t_{\text {on }}}{\Delta t_{\text {off }}}$ & $\Delta \bar{\sigma}_{t h}(\mathrm{MPa})$ & $\Delta T_{t h}(\mathrm{~K})$ & $\Delta T_{\text {end }}^{n}(\mathrm{~K})$ \\
\hline \hline$\infty$ & 40.0 & 22.2 & 17.9 \\
$1 / 5$ & 22.5 & 13.8 & 16.6 \\
$1 / 15$ & 15.0 & 6.7 & 6.4
\end{tabular}

Table 4: Fit parameters of mechanical stress relaxation after the end of deposition for continuous and interrupted growths of a $h_{f}=40 \mathrm{~nm}$ thick film. See text for definitions.

The final stress relaxation amplitude of the obtained film (Fig. 11-b) is reduced by a factor 2 for $\Delta t_{o f f}=5 \mathrm{~s}$ and 2.7 for $\Delta t_{o f f}=15 \mathrm{~s}$ compared to continuous deposition. However, the sequential stops and the corresponding short relaxations reduce the amplitudes $\Delta \bar{\sigma}_{g b}$ and $\Delta \bar{\sigma}_{\text {surf }}$ in a ratio that is more important for the fastest effect, namely the GB mechanism (Fig. 11-c,d). The correlation between relaxation amplitude and density of GBs is in line with the observations of Flötotto et al. [19], who found that the relaxation amplitude is inversely proportional to the grain size. Interruptions correspond somehow to a reduction of the average growth rate, the effect of which, as observed with evaporation, is to favour grain growth. The intrinsic characteristic times $\tau_{g b}$ and $\tau_{\text {surf }}$ are pretty similar for the three growth procedures, showing that the underlying temporal dynamics are poorly dependent on the grain microstructure and surface, as long as the film is percolated (see below Section 5).

\section{Stress relaxation in non-percolated films}

Up to now, only the stress relaxation of continuous films $\left(h_{f}=40 \mathrm{~nm}\right)$ has been scrutinized leading to nearly constant characteristic times. This section is dedicated to the study of relaxation at intermediate thicknesses of 5,9 and $20 \mathrm{~nm}$, corresponding to different morphologies of the final film $[1,5,71]$ but obtained at similar deposition conditions (Fig. 13-a). At $5 \mathrm{~nm}$, the film is beyond the compression peak (Fig. 1-a) and film consists of isolated islands in the regime of late coalescence. At $9 \mathrm{~nm}$, the film is in-between the percolation threshold and the formation of a continuous layer (Fig. 1-a). The $20 \mathrm{~nm}$ film corresponds to a continuous and homogeneous layer slightly thinner than the reference one studied up to now $\left(h_{f}=40 \mathrm{~nm}\right)$. The stress measurements show a clear increase of the final relaxation amplitude with thickness (Fig. 13). All curves could be fitted with two $\left(h_{f}=5 \mathrm{~nm}\right)$ or three $\left(h_{f}=20 ; 40 \mathrm{~nm}\right)$ exponential terms, except that of the $h_{f}=9 \mathrm{~nm}$ thick film that displays an unusual maximum.

At a thickness of $5 \mathrm{~nm}$, the thermal stress can not be reasonably included in the fit because its amplitude is small $\left(\Delta T_{\text {end }} \simeq 2 \mathrm{~K}\right)$. At the same time, relaxation phenomena are faster at this stage, given that the discontinuous film is quite unstable. Since the GBs are not fully developed and grains are closer to an 

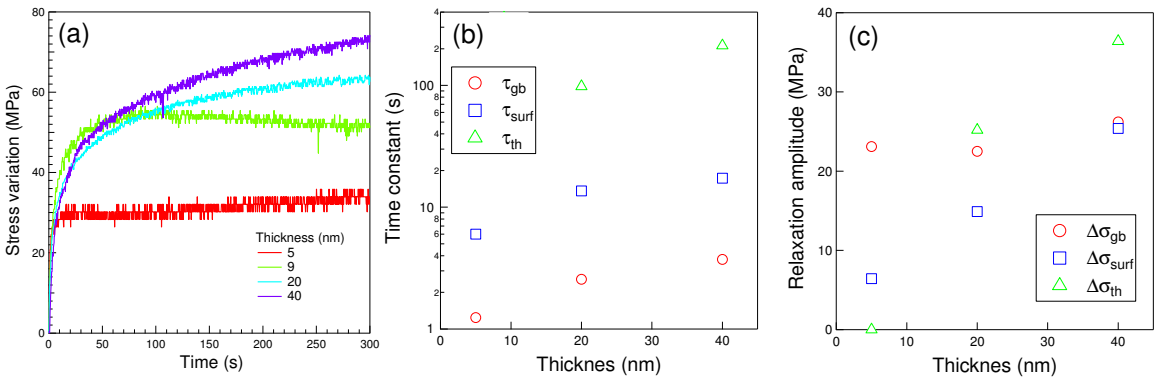

Figure 13: Stress relaxation curves of films having different final thicknesses $(P=50 \mathrm{~W}$, $p=2 \mu$ bar). Corresponding fitted (a) time constants and (b) amplitudes, obtained using two or three exponential components.

equilibrium shape at this stage of the growth, GB grooving is less effective. This hypothesis is supported by the much smaller $\Delta \bar{\sigma}_{\text {surf } f}$ value reported in Fig. 13-c. On the other hand, $\Delta \bar{\sigma}_{g b}$ is poorly affected by the initial thickness of the film, despite an increase of a change of GB height. Assuming a single grain across the film thickness, this finding implies that the out-diffusion of atoms does not involve the entire depth of the GB. As put forward in Section 3.7, a likely hypothesis is a kinetic limitation of the atom diffusion along the GBs, but not from the GBs to the surface. The smallest explored thickness sets an upper bound for the corresponding diffusion coefficient $h_{f}^{2} / \tau_{g b}=2.510^{-17} \mathrm{~m}^{2} . \mathrm{s}^{-1}$, in close agreement with the values reported in the literature [67], $D_{g b}=1.110^{-17} \mathrm{~m}^{2} . \mathrm{s}^{-1}$. Phenomena at 20 and $40 \mathrm{~nm}$ thicknesses are similar in terms of time constants. If the increase of $\Delta \bar{\sigma}_{t h}$ is the sign of an enhanced heating at $40 \mathrm{~nm}$, the increase of $\Delta \bar{\sigma}_{\text {surf }}$ (as already shown in the case of gold [20]) is explained by a smoother surface at $40 \mathrm{~nm}$, which is more prone to grooving. Finally, at $9 \mathrm{~nm}$ thickness, the slight compression that happens after the initial relaxation (above $t=100 \mathrm{~s}$; Fig. 13) remains puzzling since it does not appear at lower thickness. The last holes present in this close-to-percolation layer might be zones with a high chemical potential that favor new atom insertion.

\section{Conclusion}

Stress relaxation has been analysed in continuous Ag films deposited by direct-current sputtering at various powers and pressures. Its evolution could be fitted using three exponential terms with characteristic times spanning three orders of magnitude (from $1 \mathrm{~s}$ to $10^{2} \mathrm{~s}$ ). To clearly identify the term related to thermal stress, heating during growth and cooling during relaxation were monitored by thermocouple measurements and analysed with the help of a calorimetric model. Through gas conduction, high pressure has been found to favour slower heating and faster cooling. The increase of power, by contrast, has little effect on the final temperature because the heat flux is compensated by the decrease of deposition time for a fixed final thickness. A nearly constant deposited 
energy per atom is reported for $\mathrm{Ag}\left(15 \mathrm{eV} \cdot \mathrm{atom}^{-1}\right)$ upon varying power or pressure. For the thermal stress, similar characteristic times (around $200 \mathrm{~s}$ ) and temperature rise (around $20 \mathrm{~K}$ ), determined from stress variation amplitude, were obtained for both continuous growth and interrupted deposition. Based on a comparison between continuous/interrupted sputtering, evaporation, (thin) discontinuous film relaxation and Mo deposition, the two other exponential components of the stress relaxation dynamics were assigned to the out-diffusion of atoms from GB $\left(\tau_{g b} \simeq 3 \mathrm{~s}\right)$ and to a change of the shapes of grains at the surface via GB grooving $\left(\tau_{\text {surf }} \simeq 20 \mathrm{~s}\right)$. Their interplay was theoretically analysed in the framework of Chason's kinetic model of stress evolution. The existence of corresponding distinct exponential stress relaxation components was rationalized. In agreement with modelling, only the amplitude of the GB mechanism scales linearly with the steady-state stress during growth. However, as confirmed by the order of magnitude of the diffusion coefficient, the obtained slope and the nearly constant relaxation time point at a kinetic limitation of the diffusion of atoms along the GBs. GB grooving likely favours the release of stress in buried layers, a fact that appears rather as a consequence of surface diffusion than as its driving force. The present study demonstrates the existence of several channels of stress relaxation in metallic thin films and paves the way to their detailed analysis in many different deposition conditions.

\section{Acknowledgements}

Q.H. benefited from a PhD grant funded by Saint-Gobain Research Paris and "Association Nationale de la Recherche et de la Technologie". The technical assistance of J.F. Crepet is warmly acknowledged. This work was supported by the French National Research Agency (project FRAXOS, ANR-15-CHIN-0003).

\section{References}

[1] G. Abadias, E. Chason, J. Keckes, M. Sebastiani, G. B. Thompson, E. Barthel, G. L. Doll, C. E. Murray, C. H. Stoessel, L. Martinu, Review article: Stress in thin films and coatings: Current status, challenges, and prospects, J. Vac. Sci. Technol., A 36 (2) (2018) 020801. doi:10.1116/1.5011790.

[2] R. Koch, The intrinsic stress of polycrystalline and epitaxial thin metal films, J. Phys.: Condens. Matter 6 (1994) 9519.

[3] F. Spaepen, Interfaces and stresses in thin films, Acta Mater. 48 (2000) 31 - 42. doi:https://doi.org/10.1016/S1359-6454(99)00286-4.

[4] R. Koch, Stress in evaporated and sputtered thin films - a comparison, Surf. Coat. Technol. $204 \quad$ (2010) 1973 - 1982. doi:https://doi.org/10.1016/j.surfcoat.2009.09.047. 
[5] E. Chason, P. R. Guduru, Tutorial: understanding residual stress in polycristalline thin films through real-time measurements and physical models, J. Appl. Phys. 119 (2016) 191101.

[6] R. C. Cammarata, T. M. Trimble, D. J. Srolovitz, Surface stress model for intrinsic stresses in thin films, J. Mater. Res. 15 (2000) 2468-2474. doi:10.1557/JMR.2000.0354.

[7] R. W. Hoffman, Stresses in thin films: The relevance of grain boundaries and impurities, Thin Solid Films 34 (1976) 185-190. doi:https://doi.org/10.1016/0040-6090(76)90453-3.

[8] W. D. Nix, B. M. Clemens, Crystallite coalescence: a mechanism for intrinsic tensile stresses in thin films, J. Mater. Res. 14 (1999) 3467-3473. doi:10.1557/JMR.1999.0468.

[9] J. A. Thornton, The microstructure of sputter-deposited coatings, J. Vac. Sci. Technol., A 4 (1986) 3059-3065.

[10] S. Mahieu, P. Ghekiere, D. Depla, R. De Gryse, Biaxial alignment in sputter deposited thin films, Thin Solid Films 515 (2006) 1229-1249. doi:https://doi.org/10.1016/j.tsf.2006.06.027.

[11] D. Depla, B. R. Braeckman, Quantitative correlation between intrinsic stress and microstructure of thin films, Thin Solid Films 604 (2016) 90 - 93. doi:https://doi.org/10.1016/j.tsf.2016.03.039.

[12] C. Friesen, C. V. Thompson, Reversible stress relaxation during precoalescence interruptions of Volmer-Weber thin film growth, Phys. Rev. Lett. 89 (2002) 126103. doi:10.1103/PhysRevLett.89.126103.

[13] C. Friesen, S. C. Seel, C. V. Thompson, Reversible stress changes at all stages of Volmer-Weber film growth, J. Appl. Phys. 95 (2004) 1011-1020. doi:10.1063/1.1637728.

[14] E. Chason, B. W. Sheldon, L. B. Freund, J. A. Floro, S. J. Hearne, Origin of compressive residual stress in polycrystalline thin films, Phys. Rev. Lett. 88 (2002) 156103. doi:10.1103/PhysRevLett.88.156103.

[15] E. Chason, A kinetic analysis of residual stress evolution in polycrystalline thin films, Thin Solid Films 526 (2012) 1 - 14. doi:https://doi.org/10.1016/j.tsf.2012.11.001.

[16] A. Saedi, M. J. Rost, Thermodynamics of deposition flux-dependent intrinsic film stress, Nat. Commun. 7 (2016) 10733. doi:10.1038/ncomms10733.

[17] R. Koch, D. Hu, A. K. Das, Compressive stress in polycrystalline Volmer-Weber films, Phys. Rev. Lett. 94 (2005) 146101. doi:10.1103/PhysRevLett.94.146101. 
[18] H. Z. Yu, C. V. Thompson, Correlation of shape changes of grain surfaces and reversible stress evolution during interruptions of polycrystalline film growth, Appl. Phys. Lett. 104 (2014) 141913.

[19] D. Flötotto, Z. M. Wang, L. P. H. Jeurgens, E. J. Mittemeijer, Kinetics and magnitude of the reversible stress evolution during polycrystalline film growth interruptions, J. Appl. Phys. 118 (2015) 055305. doi:10.1063/1.4928162.

[20] H. Z. Yu, J. S. Leib, S. T. Boles, C. V. Thompson, Fast and slow stress evolution mechanisms during interruptions of Volmer-Weber growth, J. Appl. Phys. 115 (2014) 043521.

[21] C. V. Thompson, Solid-state dewetting of thin films, Annu. Rev. Mater. Res. 42 (2012) 399-434.

[22] E. Vasco, C. Polop, Comment on Correlation of shape changes of grain surfaces and reversible stress evolution during interruptions of polycrystalline film growth [Appl. Phys. Lett. 104, 141913 (2014)], Appl. Phys. Lett. 105 (2014) 246101. doi:10.1063/1.4903863.

[23] H. Z. Yu, C. V. Thompson, Response to Comment on Correlation of shape changes of grain surfaces and reversible stress evolution during interruptions of polycrystalline film growth [Appl. Phys. Lett. 105, 246101 (2014)], Appl. Phys. Lett. 105 (2014) 246102. arXiv:https://doi.org/10.1063/1.4903864, doi:10.1063/1.4903864.

[24] I. Gozhyk, L. Dai, Q. Hérault, R. Lazzari, S. Grachev, Plasma emission correction in reflectivity spectroscopy during sputtering deposition, J. Phys. D: Appl. Phys. 52 (2018) 095202. doi:10.1088/1361-6463/aaf494.

[25] G. G. Stoney, C. A. Parsons, The tension of metallic films deposited by electrolysis, Proceedings of the Royal Society of London. Series A, Containing Papers of a Mathematical and Physical Character 82 (1909) 172-175. doi:10.1098/rspa.1909.0021.

[26] http://www.knowledgedoor.com/.

[27] K. Van Aeken, D. Mahieu, D. Depla, The metal flux from a rotating cylindrical magnetron: a Monte Carlo simulation, J. Phys. D: Appl. Phys. 41 (2008) 205307. doi:10.1088/0022-3727/41/20/205307.

[28] http://draftugentbe.webhosting.be/index.php?p=137.

[29] Q. Hérault, Vers la compréhension de la croissance des couches minces d'argent par pulvérisation à la lumière de mesures operando, Ph.D. thesis, Sorbonne University (2019).

[30] F. M. d'Heurle, J. M. E. Harper, Note on the origin of intrinsic stresses in films deposited via evaporation and sputtering, Thin Solid Films 171 (1989) 81-92. 
[31] H. Windischmann, An intrinsic stress scaling law for polycrystalline thin films prepared by ion beam sputtering, J. Appl. Phys. 62 (1987) 1800-1807. doi:10.1063/1.339560.

[32] R. Abermann, H. P. Martinz, R. Kramer, Thermal effects during the deposition of thin silver, gold and copper films and their influence on internal stress measurements, Thin Solid Films 70 (1980) 127-137. doi:https://doi.org/10.1016/0040-6090(80)90420-4.

[33] L. B. Freund, S. Suresh, Thin film materials: stress, defect formation and surface evolution, Cambridge University Press, 2004.

[34] R. Koch, D. Winau, A. Führmann, K. H. Rieder, Growth-mode-specific intrinsic stress of thin silver films, Phys. Rev. B 44 (1991) 3369-3372. doi:10.1103/PhysRevB.44.3369.

[35] R. Koch, R. Abermann, On the influence of thermal effects on internal stress measurements during and after deposition of silver, gold and copper films, Thin Solid Films 129 (1985) 63-70.

[36] J. A. Thornton, Substrate heating in cylindrical magnetron sputtering sources, Thin Solid Films 54 (1978) 23-31. doi:https://doi.org/10.1016/0040-6090(78)90273-0.

[37] J. A. Thornton, J. L. Lamb, Substrate heating rates for planar and cylindrical-post magnetron sputtering sources, Thin Solid Films 119 (1984) 87-95. doi:https://doi.org/10.1016/0040-6090(84)90160-3.

[38] R. Wendt, K. Ellmer, K. Wiesemann, Thermal power at a substrate during $\mathrm{ZnO}$ :Al thin film deposition in a planar magnetron sputtering system, J. Appl. Phys. 82 (1997) 2115-2122. doi:10.1063/1.366092.

[39] H. Kersten, G. M. W. Kroesen, R. Hippler, On the energy influx to the substrate during sputter deposition of thin aluminium films, Thin Solid Films 332 (1) (1998) 282-289. doi:https://doi.org/10.1016/S0040-6090(98)010670 .

[40] S. D. Ekpe, S. K. Dew, Investigation of thermal flux to the substrate during sputter deposition of aluminum, J. Vac. Sci. Technol., A 20 (2002) 18771885. doi:10.1116/1.1507342.

[41] S. D. Ekpe, S. K. Dew, Theoretical and experimental determination of the energy flux during magnetron sputter deposition onto an unbiased substrate, J. Vac. Sci. Technol., A 21 (2003) 476-483.

[42] H. Kersten, H. Deutsch, H. Steffen, G. M. W. Kroesen, R. Hippler, The energy balance at substrate surfaces during plasma processing, Vacuum 63 (2001) 385-431. 
[43] D. Depla, S. Mahieu, Reactive Sputter Deposition, Springer, Berlin, Heidelberg, 2008. doi:https://doi.org/10.1007/978-3-540-76664-3.

[44] M. Laugier, An analysis of substrate bending during evaporation of thin films due to a small temperature difference between the substrate faces, Thin Solid Films 66 (1980) L11 - L14. doi:https://doi.org/10.1016/00406090(80)90230-8.

[45] M. Laugier, The construction and use of thin film thermocouples for the measurement of surface temperature: Applications to substrate temperature determination and thermal bending of a cantilevered plate during film deposition, Thin Solid Films 67 (1980) 163 - 170. doi:https://doi.org/10.1016/0040-6090(80)90300-4.

[46] M. V. Belous, C. M. Wayman, Temperature changes in thin metal films during vapor deposition, J. Appl. Phys. 38 (1967) 5119-5124. doi:10.1063/1.1709287.

[47] L. R. Shaginyan, V. R. Shaginyan, J. G. Han, Heating of condensation surface during magnetron sputtering, The European Physical Journal B 46 (2005) 335-342. doi:10.1140/epjb/e2005-00258-4.

[48] L. R. Shaginyan, J. G. Han, V. R. Shaginyan, J. Musil, Evolution of film temperature during magnetron sputtering, J. Vac. Sci. Technol., A 24 (4) (2006) 1083-1090. doi:10.1116/1.2210947.

[49] L. R. Shaginyan, Y. J. Kim, J. G. Han, N. V. Britun, J. Musil, I. V. Belousov, Novel model for film growth based on surface temperature developing during magnetron sputtering, Surf. Coat. Technol. 202 (2007) 486-493. doi:https://doi.org/10.1016/j.surfcoat.2007.06.016.

[50] E. Chason, A. M. Engwall, Z. Rao, T. Nishimura, Kinetic model for thin film stress including the effect of grain growth, J. Appl. Phys. 123 (2018) 185305. doi:10.1063/1.5030740.

[51] E. Chason, J. W. Shin, S. J. Hearne, L. B. Freund, Kinetic model for dependence of thin film stress on growth rate, temperature, and microstructure, J. Appl. Phys. 111 (8) (2012) 083520. doi:10.1063/1.4704683.

[52] E. Chason, M. Karlson, J. Colin, D. Magnfält, K. Sarakinos, G. Abadias, A kinetic model for stress generation in thin films grown from energetic vapor fluxes, J. Appl. Phys. 119 (14) (2016) 145307. doi:10.1063/1.4946039.

[53] D. Magnfält, A. Fillon, R. D. Boyd, U. Helmersson, K. Sarakinos, G. Abadias, Compressive intrinsic stress originates in the grain boundaries of dense refractory polycrystalline thin films, J. Appl. Phys. 119 (2016) 055305. doi:10.1063/1.4941271. 
[54] A. González-González, C. Polop, E. Vasco, Postcoalescence evolution of growth stress in polycrystalline films, Phys. Rev. Lett. 110 (2013) 056101. doi:10.1103/PhysRevLett.110.056101.

URL https://link.aps.org/doi/10.1103/PhysRevLett.110.056101

[55] E. B. Presland, G. L. Price, D. L. Trimm, The role of microstructure and surface energy in hole growth and island formation in thin silver films, Surf. Sci. 29 (1972) 435-446.

[56] H. C. Kim, T. L. Alford, D. R. Allee, Thickness dependence on the thermal stability of silver thin films, Appl. Phys. Lett. 81 (2020) 4287.

[57] P. Jacquet, R. Podor, J. Ravaux, J. Teisseire, I. Gozhyk, J. Jupille, R. Lazzari, Grain growth: the key to understand solid-state dewetting of silver thin films, Scr. Mater. 115 (2016) 128 - 132. doi:https://doi.org/10.1016/j.scriptamat.2016.01.005.

[58] P. Jacquet, R. Podor, J. Ravaux, J. Lautru, J. Teisseire, I. Gozhyk, J. Jupille, R. Lazzari, On the solid-state dewetting of polycrystalline thin films: capillary versus grain growth approach, Acta Mater. 143 (2018) 281 - 290. doi:https://doi.org/10.1016/j.actamat.2017.08.070.

[59] S. Grachev, M. De Grazia, E. Barthel, E. Søndergård, R. Lazzari, Real time monitoring of nanoparticle film growth at high deposition rate with optical spectroscopy of plasmon resonances, J. Phys. D: Appl. Phys. 46 (2013) 375305-375315.

[60] R. Lazzari, F. Leroy, G. Renaud, J. Jupille, Self-similarity during growth of the $\mathrm{Au} / \mathrm{TiO}_{2}(110)$ model catalyst as seen by grazing incidence X-ray scattering techniques, Phys. Rev B. 76 (2007) 125412.

[61] R. Lazzari, J. Jupille, Quantitative analysis of nanoparticle growth through plasmonics, Nanotechnology 22 (2011) 445703.

[62] R. Lazzari, J. Jupille, Growth kinetics and size-dependent wetting of Ag/ $\alpha$ $\mathrm{Al}_{2} \mathrm{O}_{3}(0001)$ nanoparticles studied via the plasmonic response, Nanotechnology 23 (2012) 135707.

[63] A. Debelle, G. Abadias, A. Michel, C. Jaouen, V. Pelosin, Growth stress buildup in ion beam sputtered Mo thin films and comparative study of stress relaxation upon thermal annealing or ion irradiation, J. Vac. Sci. Technol. A 25 (2007) 1438-1448. doi:10.1116/1.2771554.

[64] P. Jagtap, E. Chason, A unified kinetic model for stress relaxation and recovery during and after growth interruptions in polycrystalline thin films, Acta Materialia 193 (2020) 202-209. doi:https://doi.org/10.1016/j.actamat.2020.04.013. 
[65] A. Jamnig, N. Pliatsikas, M. Konpan, J. Lu, T. Kehagias, A. N. Kotanidis, N. Kalfagiannis, D. V. Bellas, E. Lidorikis, J. Kovac, G. Abadias, I. Petrov, J. E. Greene, K. Sarakinos, 3D-to-2D morphology manipulation of sputter-deposited nanoscale silver films on weakly interacting substrates via selective nitrogen deployment for multifunctional metal contacts, ACS Applied Nano Materials 3 (2020) 4728-4738. doi:10.1021/acsanm.0c00736.

[66] P. Guduru, E. Chason, L. Freund, Mechanics of compressive stress evolution during thin film growth, J. Mech. Phys. Solids 51 (2003) 2127 - 2148. doi:https://doi.org/10.1016/j.jmps.2003.09.013.

[67] J. Sommer, C. Herzig, Direct determination of grain-boundary and dislocation self-diffusion coefficients in silver from experiments in type-C kinetics, J. Appl. Phys. 72 (1992) 2758-2766. doi:10.1063/1.352328.

[68] W. W. Mullins, Theory of thermal grooving, J. Appl. Phys. 28 (1957) 333.

[69] P. M. Agrawal, B. M. Rice, D. L. Thompson, Predicting trends in rate parameters for self-diffusion on FCC metal surfaces, Surf. Sci. 515 (2002) $21-35$.

[70] H. Skriver, N. Rosengaard, Surface energy and work function of elemental metals, Phys. Rev. B 46 (1992) 7157-7168.

[71] G. Abadias, L. Simonot, J. J. Colin, A. Michel, S. Camelio, D. Babonneau, Volmer-weber growth stages of polycrystalline metal films probed by in situ and real-time optical diagnostics, Appl. Phys. Lett. 107 (18) (2015) 183105. doi:10.1063/1.4935034. 